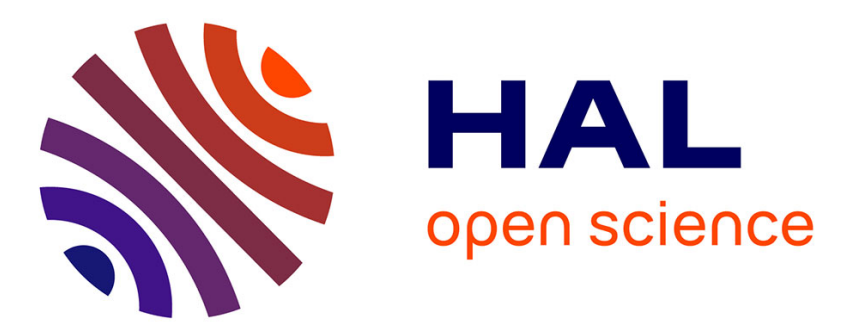

\title{
Stochastic Maximum Principle for Optimal Control ofPartial Differential Equations Driven by White Noise
}

\author{
Marco Fuhrman, Ying Hu, Gianmario Tessitore
}

\section{To cite this version:}

Marco Fuhrman, Ying Hu, Gianmario Tessitore. Stochastic Maximum Principle for Optimal Control ofPartial Differential Equations Driven by White Noise. Stochastics and Partial Differential Equations: Analysis and Computations, 2018, 6 (2), pp.255-285. 10.1007/s40072-017-0108-3 . hal-01064475v2

HAL Id: hal-01064475

https://hal.science/hal-01064475v2

Submitted on 8 Jun 2017

HAL is a multi-disciplinary open access archive for the deposit and dissemination of scientific research documents, whether they are published or not. The documents may come from teaching and research institutions in France or abroad, or from public or private research centers.
L'archive ouverte pluridisciplinaire HAL, est destinée au dépôt et à la diffusion de documents scientifiques de niveau recherche, publiés ou non, émanant des établissements d'enseignement et de recherche français ou étrangers, des laboratoires publics ou privés. 


\title{
Stochastic Maximum Principle for Optimal Control of Partial Differential Equations Driven by White Noise
}

\author{
Marco Fuhrman \\ Dipartimento di Matematica, Università di Milano \\ via Saldini 50, 20133 Milano, Italy \\ e-mail: marco.fuhrman@unimi.it \\ Ying $\mathrm{Hu}$ \\ IRMAR, Université Rennes 1 \\ Campus de Beaulieu, 35042 Rennes Cedex, France \\ e-mail: ying.hu@univ-rennes1.fr \\ Gianmario Tessitore \\ Dipartimento di Matematica e Applicazioni, Università di Milano-Bicocca \\ Via Cozzi 53, 20125 Milano, Italy \\ e-mail: gianmario.tessitore@unimib.it
}

\begin{abstract}
We prove a stochastic maximum principle of Pontryagin's type for the optimal control of a stochastic partial differential equation driven by white noise in the case when the set of control actions is convex. Particular attention is paid to well-posedness of the adjoint backward stochastic differential equation and the regularity properties of its solution with values in infinitedimensional spaces.
\end{abstract}

\section{Introduction}

In this paper we consider an infinite-dimensional stochastic optimal control problem for a system evolving in a Hilbert space $H$ and described by a state equation of the form

$$
d X_{t}=A X_{t} d t+F\left(X_{t}, u_{t}\right) d t+G\left(X_{t}, u_{t}\right) d W_{t}, \quad X_{0}=x \in H
$$

where $A$ is the infinitesimal generator of a strongly continuous semigroup $e^{t A}$ of linear operators, $W$ a cylindrical Wiener process in $H, F$ and $G$ are suitable drift and diffusion coefficients, with values in $H$ and $\mathcal{L}(H)$ respectively, depending on a control process $u$ taking values in a set $\mathcal{U}$ contained in another Hilbert space $U$. The cost function is

$$
J(x, u)=\mathbb{E} \int_{0}^{T} L\left(X_{t}, u_{t}\right) d t+\mathbb{E} \Phi\left(X_{T}\right)
$$

for suitable real-valued functions $L, \Phi$ (more precise assumptions will be given later). 
Our goal in this paper is to give a necessary condition for existence of an optimal control. This condition, called stochastic maximum principle (SMP) in the sense of Pontryagin, was extensively studied in the finite dimensional case, especially after the seminal paper by Peng [17] which gives a very general form of the SMP. There is at present a great interest in generalizations to the infinite dimensional case, that was started in [1], with particular emphasis on the application to optimal control of stochastic partial differential equations (SPDEs): see [4], [5], [6], [8], [12], [14], [15], [19], [24]. The main limitation of the present state of the art is perhaps that known results only deal with the case when the Wiener process driving the equation is finite-dimensional or has a traceclass covariance operator whereas, to our best knowledge, there is no result for a cylindrical Wiener process $W$.

In this paper our purpose is to establish the SMP for an evolution equation driven by a cylindrical Wiener process, in a form suitable for direct application to controlled SPDEs driven by white noise. On the other hand, we will suppose in this paper that the control domain $\mathcal{U}$ is convex, which allows us to apply convex perturbation arguments instead of spike variation in the deduction of the necessary optimality condition. Our main result is as follows: under suitable conditions, in particular differentiability conditions on $F, G, L, \Phi$, any optimal control $\bar{u}$ and the corresponding trajectory $\bar{X}$ must satisfy the SMP inequality

$$
\left\langle\nabla_{u}\left[F\left(\bar{X}_{t}, \bar{u}_{t}\right)\right]\left(v-\bar{u}_{t}\right), p_{t}\right\rangle+\left\langle\nabla_{u} L\left(\bar{X}_{t}, \bar{u}_{t}\right), v-\bar{u}_{t}\right\rangle+\operatorname{Tr}\left[q_{t}^{*}\left(\nabla_{u}\left[G\left(\bar{X}_{t}, \bar{u}_{t}\right)\right]\left(v-\bar{u}_{t}\right)\right)\right] \geq 0, \quad v \in \mathcal{U} .
$$

Here $\nabla$ denotes the gradient operator, $(p, q)$ is a pair of stochastic processes taking values, respectively, in $H$ and in the space of Hilbert-Schmidt operators on $H$, which we characterize as the unique solution to the so-called adjoint equation, a linear backward stochastic differential equation (BSDE) of the form

$$
\left\{\begin{array}{l}
-d p_{t}=\left[A^{*} p_{t}+\sum_{i=1}^{\infty} C_{i}^{*}(t) q_{t} e_{i}+\nabla_{x} L\left(\bar{X}_{t}, \bar{u}_{t}\right)\right] d t-\sum_{i=1}^{\infty} q_{t} e_{i} d \beta_{t}^{i}, \\
p_{T}=\nabla_{x} \Phi\left(\bar{X}_{T}\right)
\end{array}\right.
$$

where $e_{i}$ is an orthonormal basis of $H, \beta_{t}^{i}=\left\langle W_{t}, e_{i}\right\rangle$ are independent scalar Brownian motions, and $C_{i}$ are processes in $\mathcal{L}(H)$ defined as $C_{i}(t)=\nabla_{x}\left[G\left(\bar{X}_{t}, \bar{u}_{t}\right) e_{i}\right]$. There are some techincal difficulties that we have to face. The first one is the occurrence of the first series in (1.3), which needs to be interpreted in a suitable way and for which convergence holds in a weak sense in general; we call this class of BSDEs 'singular'. The second one is the occurrence of the trace operator in the SMP: for this term to be well defined we need to prove that the process $q$ satisfies additional regularity results, in particular it takes values in the space of trace-class operators on $H$, for which the trace is meaningful.

This paper is organized as follows: in the next section we introduce our control problem and formulate the main assumptions; then we prove that they are verified in the reference case of the nonlinear controlled heat equation perturbed by noise. In Section 3 we apply the convex perturbation argument, we deduce an equation for the first-order variation process and, under some more assumptions, we prove the SMP taking for granted some results on singular BSDEs. In Section 4 we make a careful study of a general class of linear singular BSDEs, proving in particular the trace-class regularity and useful duality relations. In the Appendices we conclude with some reminders and some auxiliary results. 


\section{Formulation of the Optimal Control Problem}

Given two real separable Hilbert spaces $X$ and $Y$ by $\mathcal{S}_{2}(X, Y)$ we denote the Hilbert space of Hilbert-Schmidt operators from $X$ to $Y$ and by $\mathcal{S}_{1}(X, Y)$ we denote the Banach space of trace class operators (these are instances of the Schatten-von Neumann classes of operators: some reminders are collected in Appendix 5.1). We write $\mathcal{S}_{1}(X), \mathcal{S}_{2}(X)$ instead of $\mathcal{S}_{1}(X, X), \mathcal{S}_{2}(X, X)$.

Given two Banach spaces $D$ and $E$, we say that a mapping $f: D \rightarrow E$ is of class $\mathcal{G}^{1}(D, E)$ if it is Gâteaux differentiable and $\nabla f: D \rightarrow \mathcal{L}(D, E)$ is strongly continuous (that is, continuous with respect to the strong operator topology).

Finally $H$ is a separable Hilbert space and $\left(W_{t}\right)_{t \geq 0}$ is an $H$-valued cylindrical Wiener process. We denote $\left(\mathcal{F}_{t}\right)_{t \geq 0}$ the corresponding Brownian filtration, completed in the usual way, that verifies the usual conditions.

By $L_{\mathcal{P}}^{p}([0, T] \times \Omega, H)$ we denote the Banach space of $H$-valued progressively measurable processes $X$ with $\mathbb{E} \int_{0}^{T}\left|X_{s}\right|^{p} d s<\infty$ and by $L_{\mathcal{P}}^{p}(\Omega, C([0, T], H))$ the subspace of $H$-valued progressively measurable processes $X$ with continuous trajectories satisfying $\mathbb{E} \sup _{s \in[0, T]}\left|X_{s}\right|^{p}<\infty$. Here and below we use the symbol $|\cdot|$ to denote a norm when the corresponding space is clear from the context, otherwise we use a subscript.

Assumption $2.1 \quad$ (i) $A$ is the generator of a strongly continuous semigroup $e^{t A}, t \geq 0$, of bounded linear operators in $H$.

(ii) $\mathcal{U}$ is a convex subset of a separable Banach space $U$.

(iii) $F: H \times U \rightarrow H$ is a map of class $\mathcal{G}^{1}(H \times U, H)$. Moreover, denoting its gradient on $H \times U$ as $\left(\nabla_{x} F, \nabla_{u} F\right)$, there exist constants $L, C \geq 0$ such that

$$
\left|\nabla_{x} F(x, u)\right|_{\mathcal{L}(H)}+\left|\nabla_{u} F(x, u)\right|_{\mathcal{L}(U, H)} \leq L, \quad|F(0, u)| \leq C, \quad x \in H, u \in \mathcal{U}
$$

(iv) $G: H \times U \rightarrow L(H)$ satisfies $e^{s A} G(x, u) \in \mathcal{S}_{2}(H)$ for all $s>0, x \in H, u \in U$, and the map $(x, u) \rightarrow e^{s A} G(x, u)$ is of class $\mathcal{G}^{1}\left(H \times U, \mathcal{S}_{2}(H)\right)$. Moreover there exist constants $L, C \geq 0$ and $\alpha \in[0,1 / 2)$ such that

$$
\begin{aligned}
\left|\nabla_{x}\left[e^{s A} G(x, u)\right]\right|_{\mathcal{L}\left(H, \mathcal{S}_{2}(H)\right)}+\left|\nabla_{u}\left[e^{s A} G(x, u)\right]\right|_{\mathcal{L}\left(U, \mathcal{S}_{2}(H)\right)} & \leq L s^{-\alpha} \\
\left|e^{s A} G(0, u)\right|_{\mathcal{S}_{2}(H)} & \leq C s^{-\alpha},
\end{aligned}
$$

for all $x \in H, u \in \mathcal{U}$.

(v) $L: H \times U \rightarrow \mathbb{R}$ and $\Phi: H \rightarrow \mathbb{R}$ are bounded, Lipschitz and of class $\mathcal{G}^{1}$.

(vi) There exists an orthonormal basis $\left(e_{i}\right)_{i \in \mathbb{N}} \in H$ such that, for all $i \in \mathbb{N}$ and all $u \in U$, the map $x \rightarrow G(x, u) e_{i}$ is of class $\mathcal{G}^{1}(H, H)$. Moreover there exists a constant $L \geq 0$ such that

$$
\left|\nabla_{x}\left[G(x, u) e_{i}\right]\right|_{\mathcal{L}(H)} \leq L, \quad i \in \mathbb{N}, x \in H, u \in U .
$$

(vii) For all $x, y \in H$ the map $u \rightarrow G(x, u) y$ is of class $\mathcal{G}^{1}(U, H)$ and there exists a constant $C \geq 0$ such that

$$
\left|\nabla_{u}[G(x, u) y] v\right|_{H} \leq C|y|_{H}|v|_{U}, \quad x, y \in H, u, v \in U .
$$


Any progressively measurable $\mathcal{U}$-valued process $u$ will be called an admissible control.

Under the above assumptions, for every admissible control, the state equation (formulated in mild sense):

$$
X_{t}=e^{t A} x+\int_{0}^{t} e^{(t-s) A} F\left(X_{s}, u_{s}\right) d s+\int_{0}^{t} e^{(t-s) A} G\left(X_{s}, u_{s}\right) d W_{s}
$$

admits a unique solution $X \in L_{\mathcal{P}}^{p}(\Omega, C([0, T], H))$ for every $p \geq 1$, see [2]. Moreover the cost associated to the initial datum $x$ and control $u$ is the well defined real number

$$
J(x, u)=\mathbb{E} \int_{0}^{T} L\left(X_{t}, u_{t}\right) d t+\mathbb{E} \Phi\left(X_{T}\right) .
$$

\section{Remark 2.2}

1. Combining points (iv) and (vii) of Assumption 2.1 it is easy to check that for every $s>0$, $x, h \in H, u \in U$ and any direction $v \in U$ we have

$$
\left(\nabla_{u}\left[e^{s A} G(x, u)\right] v\right) h=e^{s A} \nabla_{u}[G(x, u) h] v .
$$

Similarly, $\nabla_{x}\left[e^{s A} G(x, u)\right] e_{i}=e^{s A} \nabla_{x}\left[G(x, u) e_{i}\right]$.

2. For further use we need to introduce a suitable approximation of the derivative operator $\left(\nabla_{u}\left[e^{s A} G(x, u)\right] v\right)$. Let us denote $\Pi_{n}$ the orthogonal projection in $\mathcal{L}(H)$ onto the linear span of $e_{1}, \ldots, e_{n}$. Next, for fixed $x \in H, u, v \in U$, let us define operators in $\mathcal{L}(H)$ setting, for every $y \in H$,

$\Gamma(x, u, v) y=\nabla_{u}[G(x, u) y] v, \Gamma^{n}(x, u, v) y=\nabla_{u}\left[G(x, u)\left(\Pi_{n} y\right)\right] v=\sum_{i=1}^{n} \nabla_{u}\left[G(x, u) e_{i}\right] v\left\langle y, e_{i}\right\rangle_{H} \cdot$

Note that by $(v i)$ we have

$$
|\Gamma(x, u, v)|_{\mathcal{L}(H)} \leq C|v|, \quad\left|\Gamma^{n}(x, u, v)\right|_{\mathcal{L}(H)} \leq C|v| \sqrt{n}, \quad \Gamma^{n}(x, u, v) y \rightarrow \Gamma(x, u, v) y
$$

in $H$ as $n \rightarrow \infty$. Moreover, recalling (2.2), we have for $s>0$

$$
\left|e^{s A} \Gamma^{n}(x, u, v)-\left(\nabla_{u}\left[e^{s A} G(x, u)\right] v\right)\right|_{\mathcal{S}_{2}(H)}^{2}=\sum_{i>n}\left|e^{s A} \nabla_{u}\left[G(x, u) e_{i}\right] v\right|_{H}^{2} \downarrow 0,
$$

since the series $\sum_{i=1}^{\infty}\left|e^{s A} \nabla_{u}\left[G(x, u) e_{i}\right] v\right|_{H}^{2}=\left|\nabla_{u}\left[e^{s A} G(x, u)\right] v\right|_{\mathcal{S}_{2}(H)}^{2}$ is convergent. Finally, given a trace class operator $Q \in \mathcal{S}_{1}(H)$ it is easy to check that

$$
\left|\operatorname{Tr}\left(Q \Gamma^{n}\right)\right| \leq C|v|_{U}|Q|_{\mathcal{S}_{1}(H)}, \quad \operatorname{Tr}\left(Q \Gamma^{n}\right) \rightarrow \operatorname{Tr}(Q \Gamma) .
$$

\subsection{The Reference Example}

Consider the following controlled stochastic heat equation in $[0,1]$ :,

$$
\left\{\begin{aligned}
d X_{t}(\xi) & =\frac{\partial^{2}}{\partial x^{2}} X_{t}(\xi) d t+b\left(\xi, X_{t}(\xi), u_{t}(\xi)\right) d t+\sigma\left(\xi, X_{t}(\xi), u_{t}(\xi)\right) d \mathcal{W}(t, \xi), \\
X_{t}(0) & =X_{t}(1)=0, \quad t \in[0, T], \\
X_{0}(\xi) & =x(\xi), \quad \xi \in[0,1],
\end{aligned}\right.
$$


where $b$ and $\sigma:[0,1] \times \mathbb{R} \times \mathbb{R} \rightarrow \mathbb{R}$ are given Borel measurable functions. We assume that $b(t, \cdot, \cdot)$ and $\sigma(t, \cdot, \cdot)$ are of class $C^{1}$, Lipschitz uniformly with respect to $t$, and that $b(\cdot, 0,0)$ and $\sigma(\cdot, 0,0)$ are bounded. In the above equation $(\mathcal{W}(t, \xi)), t \geq 0, \xi \in[0,1]$ is a space time white noise and by $\left(\mathcal{F}_{t}\right)_{t \geq 0}$ we denote its natural (completed) filtration. The set of admissible control actions $\mathcal{U}$ is a convex subset of $U:=L^{2}([0,1])$ and we assume that $\mathcal{U} \subset L^{\infty}([0,1])$. A control $u$ is a (progressive) process with values in $\mathcal{U}$. We also introduce the cost functional:

$$
J(u)=\mathbb{E} \int_{0}^{T} \int_{0}^{1} l\left(\xi, X_{t}(\xi), u_{t}(\xi)\right) d \xi d t+\mathbb{E} \int_{0}^{1} h\left(\xi, X_{T}(\xi)\right) d \xi,
$$

where $l:[0,1] \times \mathbb{R} \times \mathbb{R} \rightarrow \mathbb{R}, h:[0,1] \times \mathbb{R} \rightarrow \mathbb{R}$ are given bounded, Borel measurable functions. We assume that, for a.e. $\xi \in[0,1], b(\xi, \cdot, \cdot)$ and $h(\xi, \cdot)$ are of class $C^{1}$ with bounded derivatives (uniformly with respect to $\xi$ ).

To reformulate the problem in our general framework we have set $H=L^{2}([0,1])$ and consider an $H$-valued cylindrical Wiener process $\left(W_{t}\right)_{t \geq 0}$.

$A$ is the realization of the second derivative operator in $H$ with Dirichlet boundary conditions. So $\mathcal{D}(A)=H^{2}([0,1]) \cap H_{0}^{1}([0,1])$ and $A \phi=\phi^{\prime \prime}$ for all $\phi \in \mathcal{D}(A)$.

Finally for $x, y \in L^{2}([0,1]), u, v \in L^{\infty}([0,1])$,

$$
\begin{gathered}
F(x, u)(\xi)=b(\xi, x(\xi), u(\xi)), \quad[G(x, u) y](\xi)=\sigma(\xi, x(\xi), u(\xi)) y(\xi), \\
L(x, u)=\int_{0}^{1} l(\xi, x(\xi), u(\xi)) d \xi, \quad \Phi(\xi)=\int_{0}^{1} h(\xi, X(\xi)) d \xi .
\end{gathered}
$$

The state equation written in abstract form is

$$
d X_{t}=A X_{t} d t+F\left(X_{t}, u_{t}\right) d t+G\left(X_{t}, u_{t}\right) d W_{t}, \quad X_{0}=x
$$

where $x \in H$ and the solution will evolve in $H$. Moreover the cost becomes

$$
J(x, u)=\mathbb{E} \int_{0}^{T} L\left(X_{s}, u_{s}\right) d s+\mathbb{E} \Phi\left(X_{T}\right) .
$$

It is well known (see [3]) that Assumptions $2.1(i)-(v)$ are satisfied. Concerning Assumption 2.1 (vi) we notice that in our concrete case for all $y \in L^{2}([0,1])$,

$$
\left[\nabla_{x}\left(G(x, u) e_{i}\right) y\right](\xi)=\frac{\partial \sigma}{\partial x}(\xi, x(\xi), u(\xi)) e_{i}(\xi) y(\xi) .
$$

So it is enough to choose an orthonormal basis in $L^{2}([0,1])$ with $\sup _{i} \sup _{\xi}\left|e_{i}(\xi)\right|<\infty$, for instance a trigonometrical basis.

Finally concerning Assumption 2.1 (vii) we notice that for all $y \in L^{2}([0,1])$ and all $v \in$ $L^{\infty}([0,1])$ :

$$
\left[\nabla_{u}((G(x, u) y)] v(\xi)=\frac{\partial \sigma}{\partial u}(\xi, x(\xi), u(\xi)) y(\xi) v(\xi),\right.
$$

and $\mid\left[\left.\nabla_{u}((G(x, u) y)] v(\xi)\right|_{L^{2}([0,1])} \leq L_{\sigma}|y|_{L^{2}([0,1])}|v|_{L^{\infty}([0,1])}\right.$ where $L_{\sigma}$ is the Lipschitz constant of $\sigma$ with respect to $u$. 


\section{The Stochastic Maximum Principle}

\subsection{First Variation}

In this section we perturb a given admissible control, that eventually will be the optimal one, and compute the corresponding expansion of the cost.

In the following $u$ and $u^{\prime}$ are two admissible controls and we assume that $\delta u:=u^{\prime}-u$ is bounded in $U$. Moreover we denote by $X^{\epsilon}$ the state (e.g. the solution to equation (2.1) corresponding to the control $\left.u^{\epsilon}:=(1-\epsilon) u+\epsilon u^{\prime}=u+\epsilon \delta u\right)$.

Regular dependence on parameters of the mild solution to forward SDEs gives us the first order expansion of the state:

Theorem 3.1 The map $\epsilon \rightarrow X^{\epsilon}$ is of class $\mathcal{G}^{1}$ from $[0,1)$ to $L_{\mathcal{P}}^{p}(\Omega, C([0, T], H))$ and its derivative in $\epsilon=0$ is given by the unique mild solution $Y \in L_{\mathcal{P}}^{p}(\Omega, C([0, T], H))$ of the linear equation

$$
\left\{\begin{aligned}
d Y_{t}= & {\left[A Y_{t}+\nabla_{x} F\left(X_{t}, u_{t}\right) Y_{t}+\nabla_{u} F\left(X_{t}, u_{t}\right) \delta u_{t}\right] d t } \\
& +\left[\nabla_{x} G\left(X_{t}, u_{t}\right) Y_{t}+\nabla_{u} G\left(X_{t}, u_{t}\right) \delta u_{t}\right] d W_{t}, \\
Y_{0}= & 0 .
\end{aligned}\right.
$$

Explicitly, we have $\mathbb{P}$-a.s.

$$
\begin{aligned}
Y_{t}= & \int_{0}^{t} e^{(t-s) A} \nabla_{x} F\left(X_{s}, u_{s}\right) Y_{s} d s+\int_{0}^{t} e^{(t-s) A} \nabla_{u} F\left(X_{s}, u_{s}\right) \delta u_{s} d s \\
& +\int_{0}^{t} \nabla_{x}\left[e^{(t-s) A} G\left(X_{s}, u_{s}\right)\right] Y_{s} d W_{s}+\int_{0}^{t} \nabla_{u}\left[e^{(t-s) A} G\left(X_{s}, u_{s}\right)\right] \delta u_{s} d W_{s}, \quad t \in[0, T] .
\end{aligned}
$$

Proof. The proof will follow arguments similar to the ones exposed in [7], see the proof of Proposition 3.3. We limit ourselves to proving the claim in the case $F=0$, the general case being a straightforward extension.

Consider the mapping $\Phi$ from $L_{\mathcal{P}}^{p}(\Omega, C([0, T], H)) \times[0,1)$ to $L_{\mathcal{P}}^{p}(\Omega, C([0, T], H))$ given by

$$
\Phi(\Xi, \epsilon)_{t}=e^{t A} x+\int_{0}^{t} e^{(t-l) A} G\left(\Xi_{l}, u_{l}+\epsilon \delta u_{l}\right) d W_{l} .
$$

Clearly $X^{\epsilon}$ is a solution to the state equation (2.1) with $u$ replaced by $u^{\epsilon}$ if and only if it is a fixed point of $\Phi(\cdot, \epsilon)$.

In [7] it is shown that, if $\beta>0$ is large enough then $\Phi(\cdot, \epsilon)$ is a contraction, uniformly with respect to $\epsilon$, in $L_{\mathcal{P}}^{p}(\Omega, C([0, T], H))$ endowed with the equivalent norm $\left(\mathbb{E} \sup _{t \in[0, T]} e^{\beta t}\left|\Xi_{t}\right|^{p}\right)^{1 / p}$. Moreover $\Phi(\cdot, \epsilon)$ is of class $\mathcal{G}^{1}$ from $L_{\mathcal{P}}^{p}(\Omega, C([0, T], H))$ to $L_{\mathcal{P}}^{p}(\Omega, C([0, T], H))$ with derivative, in the direction $N$, given by:

$$
\left(\nabla_{\Xi} \Phi(\Xi, \epsilon) N\right)_{t}=\int_{0}^{t} e^{(t-l) A} \nabla_{x} G\left(\Xi_{l}, u_{l}+\epsilon \delta u_{l}\right) N_{l} d W_{l} .
$$

Concerning the dependence on $\epsilon$ we have

$$
\begin{aligned}
I_{t}^{h}: & =\frac{\Phi(\Xi, \epsilon+h)_{t}-\Phi(\Xi, \epsilon)_{t}}{h}-\int_{0}^{t} \nabla_{u}\left(e^{(t-l) A} G\left(\Xi_{l}, u_{l}\right) \delta u_{l}\right) d W_{l} \\
& =\int_{0}^{t}\left\{\int_{0}^{1}\left[\nabla_{u}\left(e^{(t-l) A} G\left(\Xi_{l}, u_{l}^{\epsilon}+\zeta h \delta u_{l}\right)\right) \delta u_{l}-\nabla_{u}\left(e^{(t-l) A} G\left(\Xi_{l}, u_{l}^{\epsilon}\right)\right) \delta u_{l}\right] d \zeta\right\} d W_{l} .
\end{aligned}
$$


By the factorization method (see the proof of Proposition 3.2 in [7]) we get for $1 / p<\gamma<1 / 2-\alpha$ :

$$
\left|I^{h}\right|_{L_{\mathcal{P}}^{p}(\Omega, C([0, T], H))}^{p} \leq c_{p} \mathbb{E} \int_{0}^{T}\left|V_{l}^{h}\right|^{p} d l,
$$

where

$V_{l}^{h}=\int_{0}^{l}(l-\sigma)^{-\gamma}\left\{\int_{0}^{1}\left[\nabla_{u}\left(e^{(l-\sigma) A} G\left(\Xi_{\sigma}, u_{\sigma}^{\epsilon}+\zeta h \delta u_{\sigma}\right)\right) \delta u_{\sigma}-\nabla_{u}\left(e^{(l-\sigma) A} G\left(\Xi_{\sigma}, u_{\sigma}^{\epsilon}\right)\right) \delta u_{\sigma}\right] d \zeta\right\} d W_{\sigma}$.

By the Burkholder-Davis-Gundy inequality

$$
\begin{aligned}
\mathbb{E}\left|V_{l}^{h}\right|^{p} \leq c_{p} \mathbb{E}\left\{\int_{0}^{l}(l-\sigma)^{-2 \alpha}\right. & {\left[\int_{0}^{1} \mid \nabla_{u}\left(e^{(l-\sigma) A} G\left(\Xi_{\sigma}, u_{\sigma}^{\epsilon}+\zeta h \delta u_{\sigma}\right)\right) \delta u_{\sigma}\right.} \\
& \left.\left.-\left.\nabla_{u}\left(e^{(l-\sigma) A} G\left(\Xi_{\sigma}, u_{\sigma}^{\epsilon}\right)\right) \delta u_{\sigma}\right|_{\mathcal{S}_{2}(K, H)} ^{2} d \zeta\right] d \sigma\right\}^{p / 2} .
\end{aligned}
$$

Thus by Hypothesis 2.1-(iv):

$$
\mathbb{E}\left|V_{l}^{h}\right|^{p} \leq c_{p}\left\{|\delta u|_{L^{\infty}(\Omega \times[0, T], U)}^{2} \int_{0}^{l}(l-\sigma)^{-2(\gamma+\alpha)} d \sigma\right\}^{p / 2} \leq c_{p} l^{p / 2-p(\gamma+\alpha)}|\delta u|_{L^{\infty}(\Omega \times[0, T], U)}^{p} .
$$

The continuity of $\nabla_{u}\left(e^{(s-\sigma) A} G(\Xi, \cdot)\right)$ and Dominated Convergence Theorem yield: $\mathbb{E}\left|Y_{s}^{h}\right|^{p} \rightarrow 0$ and consequently that $\left|I^{h}\right|_{L_{\mathcal{P}}^{p}(\Omega, C([0, T], H))} \rightarrow 0$. We can therefore conclude that $\Phi$ is differentiable with respect to $\epsilon$ as well, with

$$
\nabla_{\epsilon} \Phi(\Xi, \epsilon)=\int_{0}^{t} \nabla_{u}\left(e^{(t-l) A} G\left(\Xi_{l}, u_{l}\right)\right) \delta u_{l} d W_{l} .
$$

The continuity of $\nabla_{\Xi} \Phi(\Xi, \epsilon)$ and $\nabla_{\epsilon} \Phi(\Xi, \epsilon)$ with respect to $\Xi$ and $\epsilon$ can be proved in a similar way.

Summing up, $\Phi$ is a mapping of class $\mathcal{G}^{1}$ on $L_{\mathcal{P}}^{p}(\Omega, C([0, T], H)) \times[0,1)$. The parameter depending contraction principle (see [7] Proposition 2.4) yields that the map $[0,1) \ni \epsilon \rightarrow X^{\epsilon} \in$ $L_{\mathcal{P}}^{p}(\Omega, C([0, T], H))$ that relates the parameter to the fixed point is of class $\mathcal{G}^{1}$ (in this case just differentiable with continuous derivative). Moreover its derivative satisfies:

$$
\nabla_{\epsilon} X^{\epsilon}=\nabla_{\Xi} \Phi\left(X^{\epsilon}, \epsilon\right) \nabla_{\epsilon} X^{\epsilon}+\nabla_{\epsilon} \Phi\left(X^{\epsilon}, \epsilon\right) .
$$

Plugging in the above relation the expressions for $\nabla_{\Xi} \Phi$ and $\nabla_{\epsilon} \Phi$ we get,

$$
\nabla_{\epsilon} X_{t}^{\epsilon}=\int_{0}^{t} e^{(t-l) A} \nabla_{x} G\left(X_{l}^{\epsilon}, u_{l}+\epsilon \delta u_{l}\right) \nabla_{\epsilon} X_{l}^{\epsilon} d W_{l}+\int_{0}^{t} e^{(t-l) A} \nabla_{u} G\left(X_{l}^{\epsilon}, u_{l}+\epsilon \delta u_{l}\right) \delta u_{l} d W_{l}
$$

and the claim follows letting $\epsilon=0$ and denoting $Y_{t}$ the limit.

As a consequence of the above result we have the following expansion of the cost:

Proposition 3.2 With the above notation, we have:

$$
J\left(x, u^{\epsilon}\right)=J(x, u)+\epsilon I(\delta u)+o(\epsilon),
$$

where

$$
I(\delta u)=\mathbb{E} \int_{0}^{T}\left[\left\langle\nabla_{x} L\left(X_{t}, u_{t}\right), Y_{t}\right\rangle+\left\langle\nabla_{u} L\left(X_{t}, u_{t}\right), \delta u_{t}\right\rangle\right] d t+\mathbb{E}\left\langle\nabla_{x} \Phi\left(X_{T}\right), Y_{T}\right\rangle .
$$


Proof. By the above theorem, if $R_{t}^{\epsilon}:=\epsilon^{-1}\left[X_{t}^{\epsilon}-X_{t}-\epsilon Y_{t}\right]$ then $\left|R^{\epsilon}\right|_{L_{\mathcal{P}}^{p}(\Omega, C([0, T], H))} \rightarrow 0$. Moreover

$$
\begin{aligned}
J\left(x, u^{\epsilon}\right)-J(x, u)= & \mathbb{E} \int_{0}^{T}\left[L\left(X_{t}+\epsilon\left(Y_{t}+R_{t}^{\epsilon}\right), u_{t}+\epsilon \delta u_{t}\right)-L\left(X_{t}, u_{t}\right)\right] d t \\
& +\mathbb{E}\left[\Phi\left(X_{T}+\epsilon\left(Y_{T}+R_{T}^{\epsilon}\right)\right)-\Phi\left(X_{T}\right)\right] \\
= & \epsilon \mathbb{E} \int_{0}^{T} \int_{0}^{1} \nabla_{x} L\left(X_{t}+\lambda \epsilon\left(Y_{t}+R_{t}^{\epsilon}\right), u_{t}+\lambda \epsilon \delta u_{t}\right)\left(Y_{t}+R_{t}^{\epsilon}\right) d \lambda d t \\
& +\epsilon \mathbb{E} \int_{0}^{T} \int_{0}^{1} \nabla_{u} L\left(X_{t}+\lambda \epsilon\left(Y_{t}+R_{t}^{\epsilon}\right), u_{t}+\lambda \epsilon \delta u_{t}\right) \delta u_{t} d \lambda d t \\
& +\epsilon \mathbb{E} \int_{0}^{1} \nabla_{x} \Phi\left(X_{T}+\lambda \epsilon\left(Y_{T}+R_{T}^{\epsilon}\right)\right)\left(Y_{T}+R_{T}^{\epsilon}\right) d \lambda,
\end{aligned}
$$

taking into account the continuity and boundedness of $\nabla_{x} L, \nabla_{u} L$ and $\nabla_{x} \Phi$, applying the dominated convergence theorem it is then easy to show that

$$
\begin{aligned}
& \mathbb{E} \int_{0}^{T} \int_{0}^{1} \nabla_{x} L\left(X_{t}+\lambda \epsilon\left(Y_{t}+R_{t}^{\epsilon}\right), u_{t}+\lambda \epsilon \delta u_{t}\right) Y_{t} d \lambda d t \rightarrow \mathbb{E} \int_{0}^{T} \nabla_{x} L\left(X_{t}, u_{t}\right) Y_{t} d t, \\
& \mathbb{E} \int_{0}^{T} \int_{0}^{1} \nabla_{x} L\left(X_{t}+\lambda \epsilon\left(Y_{t}+R_{t}^{\epsilon}\right), u_{t}+\lambda \epsilon \delta u_{t}\right) R_{t}^{\epsilon} d \lambda d t \rightarrow 0, \\
& \mathbb{E} \int_{0}^{T} \int_{0}^{1} \nabla_{u} L\left(X_{t}+\lambda \epsilon\left(Y_{t}+R_{t}^{\epsilon}\right), u_{t}+\lambda \epsilon \delta u_{t}\right) \delta u_{t} d \lambda d t \rightarrow \mathbb{E} \int_{0}^{T} \nabla_{u} L\left(X_{t}, u_{t}\right) \delta u_{t} d t, \\
& \mathbb{E} \int_{0}^{1} \nabla_{x} \Phi\left(X_{T}+\lambda \epsilon\left(Y_{T}+R_{T}^{\epsilon}\right)\right) Y_{T} \rightarrow \mathbb{E} \nabla_{x} \Phi\left(X_{T}\right) Y_{T}, \\
& \mathbb{E} \int_{0}^{1} \nabla_{x} \Phi\left(X_{T}+\lambda \epsilon\left(Y_{T}+R_{T}^{\epsilon}\right)\right) R_{T}^{\epsilon} \rightarrow 0,
\end{aligned}
$$

and the proof is completed.

\subsection{Stochastic Maximum Principle}

We will prove the stochastic maximum principle under the following additional assumption:

Assumption 3.3 We have $e^{t A} \in \mathcal{S}_{2}(H)$ for all $t>0$ and there exist $c>0$ and $\alpha<1 / 2$ such that

$$
\left|e^{t A}\right|_{\mathcal{S}_{2}(H)} \leq c t^{-\alpha}, \quad t \in(0, T] .
$$

In order to state the stochastic maximum principle we assume that an optimal control $\bar{u}$ exists and we denote $\bar{X}$ is the corresponding state. Next we need to introduce the dual process $\left(p_{t}, q_{t}\right)$, with values in $H \times \mathcal{S}_{2}(H)$. To this end we fix an orthonormal basis $\left\{e_{i}\right\}_{i \in \mathbb{N}}$ in $H$ such that point (vi) in Assumption 2.1 holds and we define $C_{i}(t) h=\nabla_{x}\left[G\left(\bar{X}_{t}, \bar{u}_{t}\right) e_{i}\right] h$ for $h \in H$. Then we have

$$
\left|C_{i}(t)\right|_{\mathcal{L}(H)} \leq L .
$$

Recalling Remark 2.2-1 and taking into account Hypothesis 2.1-(iv) we also obtain

$$
\sum_{i=1}^{\infty}\left|e^{t A} C_{i}(s) h\right|^{2}=\sum_{i=1}^{\infty}\left|\nabla_{x}\left[e^{t A} G\left(\bar{X}_{s}, \bar{u}_{s}\right) e_{i}\right] h\right|^{2}=\left|\nabla_{x}\left[e^{t A} G\left(\bar{X}_{s}, \bar{u}_{s}\right)\right] h\right|_{\mathcal{S}_{2}(H)}^{2} \leq L^{2} t^{-2 \alpha}|h|_{H}^{2},
$$


for all $t>0, s \geq 0, h \in H$.

Next we introduce the adjoint equation for the unknown process $(p, q)$, written formally

$$
\begin{cases}-d p_{t} & =\left[A^{*} p_{t}+\sum_{i=1}^{\infty} C_{i}^{*}(t) q_{t} e_{i}+\nabla_{x} L\left(\bar{X}_{t}, \bar{u}_{t}\right)\right] d t-\sum_{i=1}^{\infty} q_{t} e_{i} d \beta_{t}^{i}, \\ p_{T} & =\nabla_{x} \Phi\left(\bar{X}_{T}\right),\end{cases}
$$

where $\beta_{t}^{i}=\left\langle e_{i}, W_{t}\right\rangle, i=1,2 \ldots$ is a family of independent Brownian motions. The precise notion of (mild) solution to this equation is as follows: we say that a pair $(p, q)$ with $p \in L_{\mathcal{P}}^{2}(\Omega \times[0, T], H)$, $q \in L_{\mathcal{P}}^{2}\left(\Omega \times[0, T], \mathcal{S}_{2}(H)\right)$ is a mild solution to equation (3.5) if, for any $t \in[0, T]$, we have

$$
\begin{aligned}
p_{t}= & e^{(T-t) A^{*}} \nabla_{x} \Phi\left(\bar{X}_{T}\right)+\sum_{i=1}^{\infty} \int_{t}^{T} e^{(s-t) A^{*}} C_{i}^{*}(s) Q_{s} e_{i} d s \\
& +\int_{t}^{T} e^{(s-t) A^{*}} \nabla_{x} L\left(\bar{X}_{s}, \bar{u}_{s}\right) d s-\sum_{i=1}^{\infty} \int_{t}^{T} e^{(s-t) A^{*}} Q_{s} e_{i} d \beta_{s}^{i}, \quad \mathbb{P}-\text { a.s. }
\end{aligned}
$$

where, for fixed $t$, the series $\sum_{i=1}^{\infty} \int_{t}^{T} e^{(s-t) A^{*}} C_{i}^{*}(s) Q_{s} e_{i} d s$ is required to converge weakly in the space $L^{2}\left(\Omega, \mathcal{F}_{T}, \mathbb{P}, H\right)$ [in Proposition 4.18 below we will also give additional conditions that guarantee that the series converges in a stronger sense]. We refer the reader to the next section, in particular to Definition 4.1 and Remark 4.2, for a more precise discussion of this notion of solution. There we will also prove the following result.

Proposition 3.4 Under Assumptions 2.1 and 3.3 there exists a unique solution $(p, q)$ to equation (3.5). Moreover, $q_{t} \in \mathcal{S}_{1}(H) d \mathbb{P} \otimes d t$-a.s. and

$$
\mathbb{E} \int_{0}^{T}(T-t)^{2 \alpha}\left|q_{t}\right|_{\mathcal{S}_{1}(H)}^{2} d t<\infty
$$

Proof. This follows from Theorem 4.14 and Proposition 4.15. Hypothesis 4.1, needed for these statements to hold, is verified due to Assumption 3.3 and inequalities (3.3) and (3.4).

The final ingredient in the proof of the stochastic maximum principle is a duality relation involving the first variation process $Y$ solution to equation (3.1) with $u=\bar{u}$ and $X=\bar{X}$. In the present notation this equation (to be understood in the mild form (3.2)) can be written formally as

$$
\left\{\begin{aligned}
d Y_{t}= & {\left[A Y_{t}+\nabla_{x} F\left(\bar{X}_{t}, \bar{u}_{t}\right) Y_{t}+\nabla_{u} F\left(\bar{X}_{t}, \bar{u}_{t}\right) \delta u_{t}\right] d t } \\
& +\sum_{i=1}^{\infty} C_{i}(t) Y_{t} d \beta_{t}^{i}+\sum_{i=1}^{\infty} \nabla_{u}\left[G\left(\bar{X}_{t}, \bar{u}_{t}\right) e_{i}\right] \delta u_{t} d \beta_{t}^{i} \\
Y_{0}= & 0 .
\end{aligned}\right.
$$

In the next section we will prove the following duality relation.

Proposition 3.5 With the previous assumptions and notations, suppose that $\rho:[0, T] \times \Omega \rightarrow H$ and $\Gamma:[0, T] \times \Omega \rightarrow \mathcal{S}_{2}(H)$ are progressively measurable and bounded and $\mathcal{Y}$ denotes the unique mild solution to the equation

$$
\left\{\begin{array}{l}
d \mathcal{Y}_{t}=\left[A \mathcal{Y}_{t}+\nabla_{x} F\left(\bar{X}_{t}, \bar{u}_{t}\right) \mathcal{Y}_{t}+\rho_{t}\right] d t+\sum_{i=1}^{\infty} C_{i}(t) \mathcal{Y}_{t} d \beta_{t}^{i}+\sum_{i=1}^{\infty} \Gamma_{t} e_{i} d \beta_{t}^{i} \\
\mathcal{Y}_{0}=0
\end{array}\right.
$$


Then

$$
\mathbb{E} \int_{0}^{T}\left\langle p_{t}, \rho_{t}\right\rangle d t+\mathbb{E} \int_{0}^{T}\left\langle q_{t}, \Gamma_{t}\right\rangle_{\mathcal{S}_{2}(H)} d t=\mathbb{E}\left\langle\nabla_{x} \Phi\left(\bar{X}_{T}\right), \mathcal{Y}_{T}\right\rangle+\mathbb{E} \int_{0}^{T}\left\langle\nabla_{x} L\left(\bar{X}_{t}, \bar{u}_{t}\right), \mathcal{Y}_{t}\right\rangle d t
$$

Proof. This is exactly formula (4.13) of Corollary 4.8, where we put $s=0, x=0, \gamma=0$, $\eta=\nabla_{x} \Phi\left(\bar{X}_{T}\right), f_{t}=\nabla_{x} L\left(\bar{X}_{t}, \bar{u}_{t}\right)$ and note that in this case $\tilde{\mathcal{Y}}^{\infty, M}$ coincides with the solution $\mathcal{Y}$ to (3.8).

Now we are ready to state and prove the main result of this paper.

Theorem 3.6 Suppose that Assumptions 2.1 and 3.3 hold and that an optimal pair $(\bar{u}, \bar{X})$ exists. Then for every $v \in \mathcal{U}$ we have, $d \mathbb{P} \otimes d t$-a.s.,

$$
\left\langle\nabla_{u}\left[F\left(\bar{X}_{t}, \bar{u}_{t}\right)\right]\left(v-\bar{u}_{t}\right), p_{t}\right\rangle+\left\langle\nabla_{u} L\left(\bar{X}_{t}, \bar{u}_{t}\right), v-\bar{u}_{t}\right\rangle+\operatorname{Tr}\left[q_{t}^{*}\left(\nabla_{u}\left[G\left(\bar{X}_{t}, \bar{u}_{t}\right)\right]\left(v-\bar{u}_{t}\right)\right)\right] \geq 0,
$$

where $(p, q)$ is the unique mild solution to equation (3.5).

Proof. Step 1: we prove the duality formula

$$
\begin{aligned}
& \mathbb{E} \int_{0}^{T}\left\langle p_{t}, \nabla_{u} F\left(\bar{X}_{t}, \bar{u}_{t}\right) \delta u_{t}\right\rangle d t+\mathbb{E} \int_{0}^{T} \operatorname{Tr}\left[q_{t}^{*}\left(\nabla_{u}\left[G\left(\bar{X}_{t}, \bar{u}_{t}\right)\right] \delta u_{t}\right)\right] d t \\
& =\mathbb{E}\left\langle\nabla_{x} \Phi\left(\bar{X}_{T}\right), Y_{T}\right\rangle+\mathbb{E} \int_{0}^{T}\left\langle\nabla_{x} L\left(\bar{X}_{t}, \bar{u}_{t}\right), Y_{t}\right\rangle d t,
\end{aligned}
$$

where $Y$ is the first variation process solution to (3.7).

We define

$$
\rho_{t}=\nabla_{u} F\left(\bar{X}_{t}, \bar{u}_{t}\right) \delta u_{t}, \quad \Gamma_{t} h=\nabla_{u}\left[G\left(\bar{X}_{t}, \bar{u}_{t}\right) h\right] \delta u_{t},
$$

for every $h \in H$. Since we take $\delta u$ to be a bounded process, it follows that $\rho$ is also bounded, by Assumption 2.1-(iii). Heuristically, we note that with this choice the equations (3.7) and (3.8) coincide, so that $Y=\mathcal{Y}$ and (3.10) coincides with (3.9). However such argument is not correct, as we can not directly apply Proposition 3.5 above, since $\Gamma$ is not a bounded process with values in $\mathcal{S}_{2}(H)$, so we have to revert to an approximation procedure.

Let us denote $\Pi_{n}$ the orthogonal projection in $\mathcal{L}(H)$ onto the linear span of $e_{1}, \ldots, e_{n}$ and define

$$
\Gamma_{t}^{n} h=\nabla_{u}\left[G\left(\bar{X}_{t}, \bar{u}_{t}\right)\left(\Pi_{n} h\right)\right] \delta u_{t}=\sum_{i=1}^{n} \nabla_{u}\left[G\left(\bar{X}_{t}, \bar{u}_{t}\right) e_{i}\right] \delta u_{t}\left\langle h, e_{i}\right\rangle_{H}, \quad h \in H .
$$

Each $\Gamma^{n}$ is a bounded process in $\mathcal{L}(H)$ (by Assumption 2.1-(vii) and since $\delta u$ is bounded) and since it has finite rank it is also bounded in $\mathcal{S}_{2}(H)$. Let $\mathcal{Y}^{n}$ be the unique mild solution to the equation

$$
\left\{\begin{array}{l}
d \mathcal{Y}_{t}^{n}=\left[A \mathcal{Y}_{t}^{n}+\nabla_{x} F\left(\bar{X}_{t}, \bar{u}_{t}\right) \mathcal{Y}_{t}^{n}+\rho_{t}\right] d t+\sum_{i=1}^{\infty} C_{i}(t) \mathcal{Y}_{t}^{n} d \beta_{t}^{i}+\sum_{i=1}^{\infty} \Gamma_{t}^{n} e_{i} d \beta_{t}^{i}, \\
\mathcal{Y}_{0}^{n}=0 .
\end{array}\right.
$$

We can now apply Proposition 3.5 and obtain the duality relation

$$
\mathbb{E} \int_{0}^{T}\left\langle p_{t}, \rho_{t}\right\rangle d t+\mathbb{E} \int_{0}^{T}\left\langle q_{t}, \Gamma_{t}^{n}\right\rangle_{\mathcal{S}_{2}(H)} d t=\mathbb{E}\left\langle\nabla_{x} \Phi\left(\bar{X}_{T}\right), \mathcal{Y}_{T}^{n}\right\rangle+\mathbb{E} \int_{0}^{T}\left\langle\nabla_{x} L\left(\bar{X}_{t}, \bar{u}_{t}\right), \mathcal{Y}_{t}^{n}\right\rangle d t
$$


Now we let $n \rightarrow \infty$. It is convenient at this point to recall the notation introduced in Remark 2.2-2, namely the operators $\Gamma(x, u, v)$ and $\Gamma^{n}(x, u, v)$ : indeed we have

$$
\Gamma_{t}=\Gamma\left(\bar{X}_{t}, \bar{u}_{t}, \delta u_{t}\right), \quad \Gamma_{t}^{n}=\Gamma^{n}\left(\bar{X}_{t}, \bar{u}_{t}, \delta u_{t}\right),
$$

and it follows from (2.3) that for $s>0$

$$
\left|e^{s A} \Gamma_{t}^{n}-\left(\nabla_{u}\left[e^{s A} G\left(\bar{X}_{t}, \bar{u}_{t}\right)\right] \delta u_{t}\right)\right|_{\mathcal{S}_{2}(H)}^{2}=\left|e^{s A} \Gamma^{n}\left(\bar{X}_{t}, \bar{u}_{t}, \delta u_{t}\right)-\left(\nabla_{u}\left[e^{s A} G\left(\bar{X}_{t}, \bar{u}_{t}\right)\right] \delta u_{t}\right)\right|_{\mathcal{S}_{2}(H)}^{2} \downarrow 0,
$$

$d \mathbb{P} \otimes d t$-a.s., and from (2.4) that

$$
\left|\operatorname{Tr}\left(q_{t}^{*} \Gamma_{t}^{n}\right)\right| \leq C\left|\delta u_{t}\right|_{U}\left|q_{t}\right|_{\mathcal{S}_{1}(H)} \leq c\left|q_{t}\right|_{\mathcal{S}_{1}(H)}, \quad \operatorname{Tr}\left(q_{t}^{*} \Gamma_{t}^{n}\right) \rightarrow \operatorname{Tr}\left(q_{t}^{*} \Gamma_{t}\right), \quad d \mathbb{P} \otimes d t-a . s .,
$$

since we know that $q_{t} \in \mathcal{S}_{1}(H) d \mathbb{P} \otimes d t$-a.s. Writing down the mild form of the equations for $Y$ and $\mathcal{Y}^{n}$ and substracting we obtain (compare (3.2)),

$$
\begin{aligned}
\mathcal{Y}_{t}^{n}-Y_{t}= & \int_{0}^{t} e^{(t-s) A} \nabla_{x} F\left(X_{s}, u_{s}\right)\left(\mathcal{Y}_{s}^{n}-Y_{s}\right) d s+\int_{0}^{t} \nabla_{x}\left[e^{(t-s) A} G\left(X_{s}, u_{s}\right)\right]\left(\mathcal{Y}_{s}^{n}-Y_{s}\right) d W_{s} \\
& +\int_{0}^{t}\left(e^{(t-s) A} \Gamma_{t}^{n}-\nabla_{u}\left[e^{(t-s) A} G\left(X_{s}, u_{s}\right)\right] \delta u_{s}\right) d W_{s} .
\end{aligned}
$$

It follows from (3.13) that the last integral tends to zero in $L^{2}(\Omega, \mathcal{F}, \mathbb{P}, H)$. By standard estimates it also follows that $\sup _{t \in[0, T]} \mathbb{E}\left|\mathcal{Y}_{t}^{n}-Y_{t}\right|_{H}^{2} \rightarrow 0$, so that we can pass to the limit in the right-hand side of (3.12). Next we note that

$$
\mathbb{E} \int_{0}^{T}\left\langle q_{t}, \Gamma_{t}^{n}\right\rangle_{\mathcal{S}_{2}(H)} d t=\mathbb{E} \int_{0}^{T} \operatorname{Tr}\left(q_{t}^{*} \Gamma_{t}^{n}\right) d t \rightarrow \mathbb{E} \int_{0}^{T} \operatorname{Tr}\left(q_{t}^{*} \Gamma_{t}\right) d t
$$

by dominated convergence, as it follows from (3.14) and the fact that

$$
\mathbb{E} \int_{0}^{T}\left|q_{t}\right|_{\mathcal{S}_{1}(H)} d t \leq\left(\int_{0}^{T}(T-t)^{-2 \alpha} d t\right)^{1 / 2}\left(\mathbb{E} \int_{0}^{T}(T-t)^{2 \alpha}\left|q_{t}\right|_{\mathcal{S}_{1}(H)}^{2} d t\right)^{1 / 2}<\infty
$$

by Proposition 3.4 and the assumption that $\alpha<1 / 2$. Passing to the limit in (3.12) we finish the proof of Step 1.

Step 2: conclusion. It follows from Proposition 3.2 and the duality formula of Step 1 that

$$
\begin{aligned}
J\left(x, u^{\epsilon}\right)-J(x, \bar{u})= & \epsilon \mathbb{E} \int_{0}^{T}\left\langle\nabla_{u} F\left(\bar{X}_{t}, \bar{u}_{t}\right) \delta u_{t}, p_{t}\right\rangle d t+\epsilon \mathbb{E} \int_{0}^{T}\left\langle\nabla_{u} L\left(\bar{X}_{t}, \bar{u}_{t}\right), \delta u_{t}\right\rangle d t \\
& +\epsilon \mathbb{E} \int_{0}^{T} \operatorname{Tr}\left[q_{t}^{*}\left(\nabla_{u}\left[G\left(\bar{X}_{t}, \bar{u}_{t}\right)\right] \delta u_{t}\right)\right] d t+o(\epsilon)
\end{aligned}
$$

Since $\bar{u}$ is optimal, we have $J\left(x, u^{\epsilon}\right)-J(x, \bar{u}) \geq 0$ and the proof can be concluded by standard arguments based on localization and the Lebesgue differentiation theorem, see, e.g., [17, 23]. 


\section{Singular Infinite-dimensional BSDEs}

The main purpose of this section is to give a complete proof of Propositions 3.4 and 3.5 that were used in an essential way to prove the stochastic maximum principle. They both refer to properties of the dual (backward) equation (3.5). To simplify the notation we will present our results in the case when $F=0$, the general case being essentially the same. On the other hand, we will address a class of backward equations which are otherwise more general, namely of the form

$$
\begin{cases}-d P_{t} & =\left[A^{*} P_{t}+\sum_{i=1}^{\infty} C_{i}^{*}(t) Q_{t} e_{i} d t+f_{t}\right] d t-\sum_{i=1}^{\infty} Q_{t} e_{i} d \beta_{t}^{i} \\ P_{T} & =\eta\end{cases}
$$

where $\beta_{t}^{i}=\left\langle e_{i}, W_{t}\right\rangle, i=1,2 \ldots$ is a family of independent Brownian motions, $\eta \in L^{2}\left(\Omega, \mathcal{F}_{T}, \mathbb{P}, H\right)$, $f \in L_{\mathcal{P}}^{2}(\Omega \times[0, T], H)$. The unknown process is the pair denoted $(P, Q)$ and takes values in $H \times \mathcal{S}_{2}(H)$. We will work under the following assumptions, which are assumed to hold throughout this section.

\section{Hypothesis 4.1}

1. $e^{t A}, t \geq 0$, is a strongly continuous semigroup of bounded linear operators in $H$. Moreover, $e^{t A} \in \mathcal{S}_{2}(H)$ for all $t>0$ and there exist constants $c>0$ and $\alpha \in[0,1 / 2)$ such that $\left|e^{t A}\right|_{\mathcal{S}_{2}(H)} \leq c t^{-\alpha}$ for all $t \in(0, T]$.

2. The processes $C_{i}$ are strongly progressively measurable with values in $\mathcal{L}(H)$. Moreover we have $\left|C_{i}(t)\right|_{\mathcal{L}(H)} \leq c, \quad \mathbb{P}-$ a.s. for all $t \in[0, T]$ and $i \in \mathbb{N}$.

3. $\sum_{i=1}^{\infty}\left|e^{t A} C_{i}(s) h\right|^{2} \leq c t^{-2 \alpha}|h|_{H}^{2}$ for all $t \in(0, T], s \geq 0, h \in H$.

Strongly progressively measurable processes means that they are progressively measurable with values in the space $\mathcal{L}(H)$ endowed with the Borel sets of the strong operator topology.

We notice that the sum $\sum_{i=1}^{\infty} C_{i}^{*}(t) Q_{t} e_{i}$ in equation 4.1 is not convergent in general, even when $Q_{t}$ is Hilbert-Schmidt. Moreover the semigroup does not seem to be directly helpful since under Hypothesis 4.1 it is not clear whether the sum $\sum_{i=1}^{\infty} e^{s A^{*}} C_{i}^{*}(t) Q_{t} e_{i}$ converges or not. The main result of this paper is the proof of well-posedness of such class of linear BSDEs which are driven by white noise and involve a 'singular' infinite sum.

We give the following notion of (mild) solution.

Definition 4.1 We say that a pair of processes $(P, Q)$ with $P \in L_{\mathcal{P}}^{2}(\Omega \times[0, T], H), Q \in L_{\mathcal{P}}^{2}(\Omega \times$ $\left.[0, T], \mathcal{S}_{2}(H)\right)$ is a mild solution to equation (4.1) if the following holds:

1. Denoting $S^{M}(s):=\sum_{i=1}^{M}(T-s)^{\alpha} C_{i}^{*}(s) Q_{s} e_{i}, s \in[0, T]$ then the sequence $\left(S^{M}\right)$ converges weakly in $L_{\mathcal{P}}^{2}(\Omega \times[0, T], H)$.

2. For any $s \in[0, T]$,

$$
P_{s}=e^{(T-s) A^{*}} \eta+\sum_{i=1}^{\infty} \int_{s}^{T} e^{(l-s) A^{*}} C_{i}^{*}(l) Q_{l} e_{i} d l+\int_{s}^{T} e^{(l-s) A^{*}} f_{l} d l-\sum_{i=1}^{\infty} \int_{s}^{T} e^{(l-s) A^{*}} Q_{l} e_{i} d \beta_{l}^{i}, \mathbb{P}-a . s .
$$


Remark 4.2 Notice that, for any fixed $s$, the map $g \mapsto \int_{s}^{T}(T-l)^{-\alpha} e^{(l-s) A^{*}} g_{l} d l$ is a bounded linear functional from $L_{\mathcal{P}}^{2}([0, T] \times \Omega, H)$ to $L^{2}\left(\Omega, \mathcal{F}_{T}, \mathbb{P}, H\right)$, hence weakly continuous. So if condition 1 above holds then, for all fixed $s \in[0, T]$, the sum

$$
\sum_{i=1}^{M} \int_{s}^{T} e^{(l-s) A^{*}} C_{i}^{*}(l) Q_{l} d l=\int_{s}^{T}(T-l)^{-\alpha} e^{(l-s) A^{*}} S^{M}(l) d l
$$

converges, weakly in $L^{2}\left(\Omega, \mathcal{F}_{T}, \mathbb{P}, H\right)$, to a limit that we denote $\sum_{i=1}^{\infty} \int_{s}^{T} e^{(l-s) A^{*}} C_{i}^{*}(l) Q_{l} e_{i} d l$ and that appears in (4.2).

\subsection{Linear Forward SDEs}

We will study equation (4.1) exploiting duality arguments. To this end we start by collecting precise estimates on the solutions of a suitable family of linear forward SDEs. Namely, given any starting time $s \in[0, T]$, we consider the equation on the time interval $[s, T]$ :

$$
\left\{\begin{array}{l}
d \mathcal{Y}_{t}=A \mathcal{Y}_{t} d t+\sum_{i=1}^{\infty} C_{i}(t) \mathcal{Y}_{t} d \beta_{t}^{i}+\sum_{i=1}^{\infty} C_{i}(t) \gamma_{t} d \beta_{t}^{i}+\sum_{i=1}^{\infty} \Gamma_{t} e_{i} d \beta_{t}^{i}+\rho_{t} d t \\
\mathcal{Y}_{s}=x
\end{array}\right.
$$

together with the approximating equations, for $N, M \in \mathbb{N} \cup\{\infty\}$ :

$$
\left\{\begin{array}{l}
d \tilde{\mathcal{Y}}_{t}^{N, M}=A \tilde{\mathcal{Y}}_{t}^{N, M} d t+\sum_{i=1}^{N} C_{i}(t) \tilde{\mathcal{Y}}_{t}^{N, M} d \beta_{t}^{i}+\sum_{i=1}^{M} C_{i}(t) \gamma_{t} d \beta_{t}^{i}+\sum_{i=1}^{\infty} \Gamma_{t} e_{i} d \beta_{t}^{i}+\rho_{t} d t \\
\tilde{\mathcal{Y}}_{s}^{N, M}=x .
\end{array}\right.
$$

In the above equation we always assume that $x: \Omega \rightarrow H$ is bounded and $\mathcal{F}_{s}$ measurable, $\rho, \gamma:[s, T] \times \Omega \rightarrow H$ are progressively measurable and bounded, $\Gamma:[s, T] \times \Omega \rightarrow \mathcal{S}_{2}(H)$ is also progressively measurable and bounded.

For further use we note that $\tilde{\mathcal{Y}}^{N, M}$ clearly does not depend on $M$ when $\gamma=0$.

We start from a standard estimate on this SDE. Its proof coincides with the one of Proposition 3.2 in [7] and will be omitted.

Theorem 4.3 For all $p \in[2, \infty)$ and $N, M \in \mathbb{N} \cup\{\infty\}$, in the space $L_{\mathcal{P}}^{p}(\Omega, C([s, T], H))$ there exists a unique solution $\mathcal{Y}$ to equation (4.3) and a unique solution $\tilde{\mathcal{Y}}^{N, M}$ to equation (4.4); note that $\tilde{\mathcal{Y}}^{\infty, \infty}=\mathcal{Y}$. Moreover, if $p$ is large enough, the following estimate holds:

$$
\mathbb{E}\left(\sup _{t \in[s, T]}\left|\mathcal{Y}_{t}\right|^{p}\right) \leq c_{p}\left(1+\mathbb{E}|x|^{p}+|\Gamma|_{L^{\infty}([s, T] \times \Omega, \mathcal{L}(H))}^{p}+|\gamma|_{L_{\mathcal{P}}^{\infty}([s, T] \times \Omega, H)}^{p}+\mathbb{E}\left(\int_{s}^{T}\left|\rho_{t}\right| d t\right)^{p}\right) .
$$

We also have

$$
\tilde{\mathcal{Y}}^{N, M} \rightarrow \tilde{\mathcal{Y}}^{N, \infty}, \quad \tilde{\mathcal{Y}}^{N, M} \rightarrow \tilde{\mathcal{Y}}^{\infty, M}, \quad \tilde{\mathcal{Y}}^{M, M} \rightarrow \mathcal{Y}, \quad \tilde{\mathcal{Y}}^{N, \infty} \rightarrow \mathcal{Y}, \quad \tilde{\mathcal{Y}}^{\infty, M} \rightarrow \mathcal{Y}
$$

in the norm of $L_{\mathcal{P}}^{p}(\Omega, C([s, T], H))$, namely $\mathbb{E}\left(\sup _{t \in[s, T]}\left|\tilde{\mathcal{Y}}_{t}^{N, M}-\tilde{\mathcal{Y}}_{t}^{N, \infty}\right|^{p}\right) \rightarrow 0$ etc.

In addition, the above estimate holds for $\tilde{\mathcal{Y}}^{N, M}, \tilde{\mathcal{Y}}^{N, \infty}, \tilde{\mathcal{Y}}^{\infty, M}$ uniformly with respect to $N$ and $M$.

Finally, the first (respectively, the second) convergence result in (4.5) holds true uniformly with respect to $N$ (respectively, to $M$ ). 
The next estimate involves the Hilbert-Schmidt norm of $\Gamma$.

Proposition 4.4 Under the above assumptions and notations it holds

$$
\sup _{t \in[s, T]} \mathbb{E}\left|\mathcal{Y}_{t}\right|^{2} \leq c \mathbb{E}\left[|x|^{2}+\left(\int_{s}^{T}\left|\rho_{t}\right| d t\right)^{2}+|\gamma|_{L_{\mathcal{P}}^{\infty}([s, T] \times \Omega, H)}^{2}+\int_{s}^{T}\left|\Gamma_{t}\right|_{\mathcal{S}_{2}(H)}^{2} d t\right] .
$$

Proof. Writing equation (4.3) in the mild form, namely

$$
\mathcal{Y}_{t}=e^{(t-s) A} x+\int_{s}^{t} e^{(t-l) A} \rho_{l} d l+\sum_{i=1}^{\infty} \int_{s}^{t} e^{(t-l) A}\left[C_{i}(l)\left(\mathcal{Y}_{l}+\gamma_{l}\right)+\Gamma_{l} e_{i}\right] d \beta_{l}^{i},
$$

and taking into account Hypothesis 4.1 we get

$$
\begin{aligned}
\mathbb{E}\left|\mathcal{Y}_{t}\right|^{2} \leq & c \mathbb{E}\left[|x|^{2}+\left(\int_{s}^{t}\left|\rho_{l}\right| d l\right)^{2}+\int_{s}^{t}(t-l)^{-2 \alpha}\left|\gamma_{l}\right|^{2} d l+\int_{s}^{t}\left|e^{(t-l) A} \Gamma_{l}\right|_{\mathcal{S}_{2}(H)}^{2} d l\right] \\
& +c \int_{s}^{t}(t-l)^{-2 \alpha} \mathbb{E}\left|\mathcal{Y}_{l}\right|^{2} d l .
\end{aligned}
$$

We obtain

$$
\mathbb{E}\left|\mathcal{Y}_{t}\right|^{2} \leq c \mathbb{E}\left[|x|^{2}+\left(\int_{s}^{T}\left|\rho_{l}\right| d l\right)^{2}+|\gamma|_{L^{\infty}}^{2}+\int_{s}^{T}\left|\Gamma_{l}\right|_{\mathcal{S}_{2}(H)}^{2} d l\right]+c \int_{s}^{t}(t-l)^{-2 \alpha} \mathbb{E}\left|\mathcal{Y}_{l}\right|^{2} d l
$$

and the claim then follows applying a variant of the Gronwall Lemma, see Lemma 7.1.1 in [10].

The final estimate will be an important tool in the rest of the paper and again exploits a special version of the Gronwall Lemma.

Proposition 4.5 For $s \leq t \leq T$,

$$
\mathbb{E}\left|\mathcal{Y}_{t}\right|^{2} \leq c \mathbb{E}\left[|x|^{2}+\int_{s}^{t}(t-l)^{-2 \alpha}\left|\gamma_{l}\right|_{H}^{2} d l+\int_{s}^{t}(t-l)^{-2 \alpha}\left|\Gamma_{l}\right|_{\mathcal{L}(H)}^{2} d l+\left(\int_{s}^{t}\left|\rho_{l}\right| d l\right)^{2}\right] .
$$

Proof. We first note that

$$
\left.\left|e^{(t-l) A} \Gamma_{l}\right|_{\mathcal{S}_{2}(H)}|\leq| e^{(t-l) A}\right|_{\mathcal{S}_{2}(H)}\left|\Gamma_{l}\right|_{\mathcal{L}(H)} \leq c(t-l)^{-\alpha}\left|\Gamma_{l}\right|_{\mathcal{L}(H)} .
$$

Consequently, letting

$$
u(t):=\mathbb{E}\left|\mathcal{Y}_{t}\right|^{2}, \quad v(t):=c \mathbb{E}\left(\left|\Gamma_{t}\right|_{L(H)}^{2}+\left|\gamma_{t}\right|^{2}\right), \quad w(t):=c \mathbb{E}|x|^{2}+c \mathbb{E}\left(\int_{s}^{t}\left|\rho_{l}\right| d l\right)^{2},
$$

it follows from (4.6) that

$$
u(t) \leq w(t)+\int_{s}^{t}(t-l)^{-2 \alpha} v(l) d l+c \int_{s}^{t}(t-l)^{-2 \alpha} u(l) d l=e(t)+c \int_{s}^{t}(t-l)^{-2 \alpha} u(l) d l,
$$


where we set $e(t)=w(t)+\int_{s}^{t}(t-l)^{-2 \alpha} v(l) d l$. Using again the Gronwall Lemma in [10] Lemma 7.1.1 we obtain

$$
u(t) \leq e(t)+c \int_{s}^{t}(t-l)^{-2 \alpha} e(l) d l .
$$

Next we note that

$$
\begin{aligned}
\int_{s}^{t}(t-l)^{-2 \alpha} e(l) d l & =\int_{s}^{t} w(l)(t-l)^{-2 \alpha} d l+c \int_{s}^{t}(t-l)^{-2 \alpha} \int_{s}^{l}(l-\zeta)^{-2 \alpha} v(\zeta) d \zeta \\
& \leq w(t) \int_{s}^{t}(t-l)^{-2 \alpha} d l+c \int_{s}^{t}(t-l)^{-2 \alpha} \int_{s}^{l}(l-\zeta)^{-2 \alpha} v(\zeta) d \zeta \\
& \leq c w(t)+c \int_{s}^{t} v(\zeta) \int_{\zeta}^{t}(t-l)^{-2 \alpha}(l-\zeta)^{-2 \alpha} d l d \zeta .
\end{aligned}
$$

But since

$$
\begin{aligned}
\int_{\zeta}^{t}(t-l)^{-2 \alpha}(l-\zeta)^{-2 \alpha} d l & =\int_{0}^{t-\zeta}(t-\zeta-s)^{-2 \alpha} s^{-2 \alpha} d s=\int_{0}^{1}(1-z)^{-2 \alpha} z^{-2 \alpha}(t-\zeta)^{-4 \alpha+1} d z \\
& =c(t-\zeta)^{-4 \alpha+1} \leq c(t-\zeta)^{-2 \alpha}
\end{aligned}
$$

from (4.8) we obtain the required conclusion:

$$
u(t) \leq c w(t)+c \int_{s}^{t}(t-l)^{-2 \alpha} v(l) d l .
$$

Corollary 4.6 Integrating the inequality (4.7) with respect to $t$ we get

$$
E \int_{s}^{T}\left|\mathcal{Y}_{l}\right|^{2} d l \leq c \mathbb{E}\left[|x|^{2}+\int_{s}^{T}\left(\left|\Gamma_{l}\right|_{L(H)}^{2}+\left|\gamma_{l}\right|^{2}\right) d l+\left(\int_{s}^{T}\left|\rho_{l}\right| d l\right)^{2}\right]
$$

\subsection{Existence of a Solution to the Singular BSDE}

We will proceed by approximation. Namely, we consider the following BSDE in infinite dimensions where the singular sum in the drift has been truncated:

$$
\begin{cases}-d P_{s}^{N} & =\left[A^{*} P_{s}^{N}+\sum_{i=1}^{N} C_{i}^{*}(s) Q_{t}^{N} e_{i}+f_{s}\right] d s-\sum_{i=1}^{\infty} Q_{s}^{N} e_{i} d \beta_{s}^{i} \\ P_{T}^{N} & =\eta\end{cases}
$$

We still assume that $\eta \in L^{2}\left(\Omega, \mathcal{F}_{T}, \mathbb{P}, H\right), f \in L_{\mathcal{P}}^{2}(\Omega \times[0, T], H)$, so the above equation fits in the classical theory of Hilbert valued BSDEs, see [11]. In particular it holds:

Proposition 4.7 Assume that $\eta \in L^{2}\left(\Omega, \mathcal{F}_{T}, \mathbb{P}, H\right), f \in L_{\mathcal{P}}^{2}(\Omega \times[0, T], H)$. Then there exists a unique $\left(P^{N}, Q^{N}\right)$ with

$$
P^{N} \in L_{\mathcal{P}}^{2}\left(\Omega, C([0, T], H), \quad Q^{N} \in L_{\mathcal{P}}^{2}\left(\Omega \times[0, T], \mathcal{S}_{2}(H)\right)\right.
$$


verifying (4.10) in the following mild sense:

$$
P_{s}^{N}=e^{(T-s) A^{*}} \eta+\int_{s}^{T} e^{(l-s) A^{*}}\left[\sum_{i=1}^{N} C_{i}^{*}(l) Q_{l}^{N} e_{i}+f_{l}\right] d l-\sum_{i=1}^{\infty} \int_{s}^{T} e^{(l-s) A^{*}} Q_{l}^{N} e_{i} d \beta_{l}^{i} .
$$

Moreover choosing as before $x: \Omega \rightarrow H$ bounded and $\mathcal{F}_{s}$ measurable, $\rho, \gamma$ from $[s, T] \times \Omega$ to $H$ progressively measurable and bounded and $\Gamma:[s, T] \times \Omega \rightarrow \mathcal{S}_{2}(H)$ progressively measurable and bounded it holds:

$$
\begin{aligned}
& \mathbb{E}\left\langle P_{s}^{N}, x\right\rangle+\mathbb{E} \int_{s}^{T}\left\langle P_{l}^{N}, \rho_{l}\right\rangle d l+\mathbb{E} \int_{s}^{T}\left\langle Q_{l}^{N}, \Gamma_{l}\right\rangle_{\mathcal{S}_{2}(H)} d l+\mathbb{E} \int_{s}^{T} \sum_{i=1}^{M}\left\langle Q_{l}^{N} e_{i}, C_{i}(l) \gamma_{l}\right\rangle d l \\
& =\mathbb{E}\left\langle\eta, \tilde{\mathcal{Y}}_{T}^{N, M}\right\rangle+\mathbb{E} \int_{s}^{T}\left\langle f_{l}, \tilde{\mathcal{Y}}_{l}^{N, M}\right\rangle d l,
\end{aligned}
$$

where $\tilde{\mathcal{Y}}^{N, M}$ is the solution to equation (4.4).

We now define a candidate solution $(P, Q)$ as the weak limit of $\left(P^{N}, Q^{N}\right)$ in some Hilbert space.

Corollary 4.8 We have

1. $P^{N}$ converges weakly to an element $P$ in $L_{\mathcal{P}}^{2}([0, T] \times \Omega, H)$;

2. for any $t, P_{t}^{N}$ converges weakly to an element $\tilde{P}_{t}$ in $L^{2}\left(\Omega, \mathcal{F}_{t}, \mathbb{P}, H\right)$;

3. $Q^{N}$ converges weakly to an element $Q$ in $L_{\mathcal{P}}^{2}\left([0, T] \times \Omega, \mathcal{S}_{2}(H)\right)$.

Moreover choosing $\eta, f, x, \rho, \gamma, \Gamma$ as in Proposition 4.7 it holds, for all $M \in \mathbb{N}$ :

$$
\begin{aligned}
& \mathbb{E}\left\langle\tilde{P}_{s}, x\right\rangle+\mathbb{E} \int_{s}^{T}\left\langle P_{l}, \rho_{l}\right\rangle d l+\mathbb{E} \int_{s}^{T}\left\langle Q_{l}, \Gamma_{l}\right\rangle_{\mathcal{S}_{2}(H)} d l+\mathbb{E} \int_{s}^{T} \sum_{i=1}^{M}\left\langle Q_{l} e_{i}, C_{i}(l) \gamma_{l}\right\rangle d l \\
& =\mathbb{E}\left\langle\eta, \tilde{\mathcal{Y}}_{T}^{\infty, M}\right\rangle+\mathbb{E} \int_{s}^{T}\left\langle f_{l}, \tilde{\mathcal{Y}}_{l}^{\infty, M}\right\rangle d l .
\end{aligned}
$$

Proof. Let us consider the processes $\mathcal{Y}$ and $\tilde{\mathcal{Y}}^{N, M}$, defined as solutions to equations (4.3) and (4.4). In the first part of the proof we take $s=0, x=0$ and $\gamma=0$ in these equations and we recall that the processes $\tilde{\mathcal{Y}}^{N, M}$ do not depend on $M$, so that in particular $\tilde{\mathcal{Y}}^{\infty, M}=\mathcal{Y}$.

By the estimates in Proposition 4.4 the maps

$$
\mathcal{T}:(\rho, \Gamma) \mapsto\left(\tilde{\mathcal{Y}}_{T}^{\infty, M}, \tilde{\mathcal{Y}}^{\infty, M}\right), \quad \mathcal{T}_{N}:(\rho, \Gamma) \mapsto\left(\tilde{\mathcal{Y}}_{T}^{N, M}, \tilde{\mathcal{Y}}^{N, M}\right)
$$

can be extended to bounded linear maps from the space $L_{\mathcal{P}}^{2}(\Omega \times[0, T], H) \times L_{\mathcal{P}}^{2}\left(\Omega \times[0, T], \mathcal{S}_{2}(H)\right)$ to $L^{2}\left(\Omega, \mathcal{F}_{T}, \mathbb{P}, H\right) \times L_{\mathcal{P}}^{2}(\Omega \times[0, T], H)$. We denote by $\mathcal{T}^{*}, \mathcal{T}_{N}^{*}$ their Hilbert space adjoints. Given arbitrary $\eta \in L^{2}\left(\Omega, \mathcal{F}_{T}, \mathbb{P}, H\right), f \in L_{\mathcal{P}}^{2}(\Omega \times[0, T], H)$ and setting $(P, Q)=\mathcal{T}^{*}(\eta, f)$, we see that

$$
\mathbb{E} \int_{0}^{T}\left\langle P_{l}, \rho_{l}\right\rangle d l+\mathbb{E} \int_{0}^{T}\left\langle Q_{l}, \Gamma_{l}\right\rangle_{\mathcal{S}_{2}(H)} d l=\mathbb{E}\left\langle\eta, \mathcal{Y}_{T}^{M}\right\rangle+\mathbb{E} \int_{0}^{T}\left\langle f_{l}, \mathcal{Y}_{l}^{M}\right\rangle d l,
$$

while (4.12) (with $s=0, x=0, \gamma=0$ ) shows that $\left(P^{N}, Q^{N}\right)=\mathcal{T}_{N}^{*}(\eta, f)$. Since $\tilde{\mathcal{Y}}^{N, M} \rightarrow \tilde{\mathcal{Y}}^{\infty, M}$ as specified in Theorem 4.3, it follows easily that $P^{N} \rightarrow P$ weakly in $L_{\mathcal{P}}^{2}([0, T] \times \Omega, H)$ and $Q^{N} \rightarrow Q$ weakly in $L_{\mathcal{P}}^{2}\left([0, T] \times \Omega, \mathcal{S}_{2}(H)\right)$. 
Now, for arbitrary $s \in[0, T]$ and $x \in L^{2}\left(\Omega, \mathcal{F}_{s}, \mathbb{P}, H\right)$ it follows from (4.12) (still with $\gamma=0$ ) that $\mathbb{E}\left\langle P_{s}^{N}, x\right\rangle$ has a limit as $N \rightarrow \infty$, equal to

$$
-\mathbb{E} \int_{s}^{T}\left\langle P_{l}^{N}, \rho_{l}\right\rangle d l-\mathbb{E} \int_{s}^{T}\left\langle Q_{l}^{N}, \Gamma_{l}\right\rangle_{\mathcal{S}_{2}(H)} d l+\mathbb{E}\left\langle\eta, \tilde{\mathcal{Y}}_{T}^{N, M}\right\rangle+\mathbb{E} \int_{s}^{T}\left\langle f_{l}, \tilde{\mathcal{Y}}_{l}^{N, M}\right\rangle d l .
$$

This shows that $P_{s}^{N}$ converges weakly in $L^{2}\left(\Omega, \mathcal{F}_{s}, \mathbb{P}, H\right)$, and we denote by $\tilde{P}_{s}$ its limit.

Finally, coming back to general $\gamma$, equality (4.13) follows from (4.12) letting $N \rightarrow \infty$.

We then need to get some regularity property of the process $\left(\tilde{P}_{s}\right)$ which, for the moment, we can not identify with $\left(P_{s}\right)$ and has only been defined $\mathbb{P}$-a.s. for any fixed $s \in[0, T]$.

Proposition 4.9 The map $s \mapsto \tilde{P}_{s}$ is weakly continuous from $[0, T]$ to $L^{2}\left(\Omega, \mathcal{F}_{T}, \mathbb{P}, H\right)$.

Proof. In order to stress its dependence on the initial time and state, given $s \in[0, T]$ and $x \in L^{2}\left(\Omega, \mathcal{F}_{s}, \mathbb{P}, H\right)$, denote by $\mathcal{Y}^{x, s}$ the solution of equation (4.3) with $\rho=\Gamma=\gamma=0$, namely:

$$
d \mathcal{Y}_{t}^{\xi, s}=A \mathcal{Y}_{t}^{\xi, s} d s+\sum_{i=1}^{\infty} C_{i}(s) \mathcal{Y}_{t}^{\xi, s} d \beta_{s}^{i}, \quad \mathcal{Y}_{s}^{\xi, s}=x
$$

We know by [2] that the above equation admits a unique mild solution with $\sup _{t \in[s, T]} \mathbb{E}\left|\mathcal{Y}_{t}^{x, s}\right|^{2} \leq$ $c_{T}\left(1+\mathbb{E}|x|^{2}\right)$, morever if $x^{\prime} \in L^{2}\left(\Omega, \mathcal{F}_{s}, \mathbb{P}, H\right)$ then $\sup _{t \in[s, T]} \mathbb{E}\left|\mathcal{Y}_{t}^{x, s}-\mathcal{Y}_{t}^{x^{\prime}, s}\right|^{2} \leq c_{T}\left(\mathbb{E}\left|x-x^{\prime}\right|^{2}\right)$.

For fixed $x \in L^{2}\left(\Omega, \mathcal{F}_{T}, \mathbb{P}, H\right)$, let us denote $x_{s}:=\mathbb{E}\left(x \mid \mathcal{F}_{s}\right)$. Then equation (4.13) yields:

$$
\mathbb{E}\left\langle\tilde{P}_{s}, x\right\rangle=\mathbb{E}\left\langle\tilde{P}_{s}, x_{s}\right\rangle=\mathbb{E}\left\langle\mathcal{Y}_{T}^{x_{s}, s}, \eta\right\rangle+\mathbb{E} \int_{s}^{T}\left\langle\mathcal{Y}_{l}^{x_{s}, s}, f_{l}\right\rangle d l .
$$

Since

$$
\sup _{s \in[0, T]} \sup _{t \in[s, T]} \mathbb{E}\left|\mathcal{Y}_{t}^{s, x_{s}}\right|^{2} \leq \sup _{s \in[0, T]} c_{T}\left(1+\mathbb{E}\left|x_{s}\right|^{2}\right) \leq c,
$$

the weak continuity is proved if we show that, for all $t>s$, the map $s \mapsto \mathcal{Y}_{t}^{s, x_{s}}$ is continuous in the norm of $L^{2}(\Omega, \mathcal{F}, \mathbb{P}, H)$.

If $s_{n} \downarrow s$ then $\mathbb{E}\left|\mathcal{Y}_{t}^{s_{n}, x_{s_{n}}}-\mathcal{Y}_{t}^{s, x_{s}}\right|^{2} \leq 2 \mathbb{E}\left|\mathcal{Y}_{t}^{s_{n}, x_{s_{n}}}-\mathcal{Y}_{t}^{s_{n}, x_{s}}\right|^{2}+2 \mathbb{E}\left|\mathcal{Y}_{t}^{s_{n}, x_{s}}-\mathcal{Y}_{t}^{s, x_{s}}\right|^{2}$. The first term is controlled by $\mathbb{E}\left|x_{s_{n}}-x_{s}\right|^{2}=\mathbb{E}\left|\mathbb{E}\left(x \mid \mathcal{F}_{s_{n}}\right)-\mathbb{E}\left(x \mid \mathcal{F}_{s}\right)\right|^{2} \rightarrow 0$.

The second term is $\mathbb{E}\left|\mathcal{Y}_{t}^{s_{n}, x_{s}}-\mathcal{Y}_{t}^{s_{n}, \mathcal{Y}_{s_{n}}^{s, x_{s}}}\right|^{2}$ and is controlled by

$$
\begin{aligned}
\mathbb{E}\left|\mathcal{Y}_{s_{n}}^{s, x_{s}}-x_{s}\right|^{2} & \leq 2 \mathbb{E}\left|e^{\left(s_{n}-s\right) A} x_{s}-x_{s}\right|^{2}+2 \mathbb{E}\left|\sum_{i=1}^{\infty} \int_{s}^{s_{n}} e^{\left(s_{n}-t\right) A} C_{i}(t) \mathcal{Y}_{t}^{s, x_{s}} d \beta_{t}^{i}\right|^{2} \\
& \leq 2 \mathbb{E}\left|e^{\left(s_{n}-s\right) A} x_{s}-x_{s}\right|^{2}+c \sup _{l \in[s, T]} \mathbb{E}\left|\mathcal{Y}_{l}^{s, x_{s}}\right|^{2} \int_{s}^{s_{n}}\left(s_{n}-t\right)^{-2 \alpha} d t
\end{aligned}
$$

which converges to 0 as $s_{n} \downarrow 0$.

On the other hand, if $s_{n} \uparrow s, \mathcal{Y}_{t}^{s_{n}, x_{s_{n}}}-\mathcal{Y}_{t}^{s, x}=\mathcal{Y}_{t}^{s, \mathcal{Y}_{s}^{s_{n}, x_{s_{n}}}}-\mathcal{Y}_{t}^{s, x}$. Hence it is enough to prove that $\mathcal{Y}_{s}^{s_{n}, x_{s_{n}}} \rightarrow x$. in the norm of $L^{2}(\Omega, \mathcal{F}, \mathbb{P}, H)$. But, proceeding as before, we have

$$
\mathbb{E}\left|\mathcal{Y}_{s}^{x_{s_{n}}, s_{n}}-x\right|^{2} \leq 2 \mathbb{E}\left|e^{\left(s-s_{n}\right) A} x_{s_{n}}-x\right|^{2}+c \int_{s_{n}}^{s}(s-l)^{-2 \alpha} \mathbb{E}\left|\mathcal{Y}_{l}^{x_{s_{n}}, s_{n}}\right|^{2} d l
$$


The claim follows recalling that $\sup _{n} \sup _{l \in\left[s_{n}, s\right]} \mathbb{E}\left|\mathcal{Y}^{x_{s_{n}}, s_{n}}\right|^{2} \leq c_{T} \sup _{n}\left(1+\mathbb{E}\left|x_{s_{n}}\right|^{2}\right)<\infty$ and that $\left|e^{\left(s-s_{n}\right) A} x_{s_{n}}-x\right| \leq c\left|x_{s_{n}}-x\right|+\left|e^{\left(s-s_{n}\right) A} x-x\right|$.

We are now in a position to prove that $\tilde{P}$ and $P$ coincide.

Proposition 4.10 $\tilde{P}$ is a progressively measurable process and $\tilde{P}=P, d t \otimes d \mathbb{P}$-a.s.

Proof. We first prove progressive measurability. Fixed an arbitrary $t \in[0, T]$ we choose a basis $\left\{\varphi_{m}\right\}$ in $L^{2}\left(\Omega, \mathcal{F}_{t}, \mathbb{P}, H\right)$. We have $\tilde{P}_{l}=\sum_{m=1}^{\infty}\left(\mathbb{E}\left\langle\tilde{P}_{l}, \varphi_{m}\right\rangle_{H}\right) \varphi_{m}, \forall l \leq t$. Since $\mathbb{E}\left\langle\tilde{P}_{l}, \varphi_{m}\right\rangle_{H}$ is a continuous function we immediately deduce that $\tilde{P}$ restricted to $[0, t]$ is $\mathcal{B}[0, t] \otimes \mathcal{F}_{t}$ measurable.

To show that $(\tilde{P})$ and $(P)$ coincide choose $x=\gamma=\Gamma=0$ and an arbitrary bounded progressively measurable process $\rho$ in (4.3). By definition of $\tilde{P}$, for all $t \in[0, T], \mathbb{E}\left\langle P_{t}^{N}, \rho_{t}\right\rangle \rightarrow \mathbb{E}\left\langle\tilde{P}_{t}, \rho_{t}\right\rangle$ so, by dominated convergence theorem (exploiting the measurability of $(\tilde{P}))) \int_{0}^{T} \mathbb{E}\left\langle P_{l}^{N}, \rho_{l}\right\rangle d l \rightarrow \int_{0}^{T} \mathbb{E}\left\langle\tilde{P}_{l}, \rho_{l}\right\rangle d l$. But we already know, see Corollary 4.8 , that $\int_{0}^{T} \mathbb{E}\left\langle P_{l}^{N}, \rho_{l}\right\rangle d l \rightarrow \int_{0}^{T} \mathbb{E}\left\langle P_{l}, \rho_{l}\right\rangle d l$ and the claim is proved.

In the following when we refer to the process $P$ we will always refer to its version $\tilde{P}$.

We come now to the study of process $Q$.

Lemma 4.11 Setting $S^{M}(s)=\sum_{i=1}^{M}(T-s)^{\alpha} C_{i}^{*}(s) Q_{s} e_{i}, S^{M}$ converges weakly in $L_{\mathcal{P}}^{2}(\Omega \times[0, T] ; H)$. The limit will be denoted by $\sum_{i=1}^{\infty}(T-\cdot)^{\alpha} C_{i}^{*}(\cdot) Q . e_{i}$.

Proof. Given an arbitrary bounded progressively measurable process $\gamma$ in $H$, let $\hat{\mathcal{Y}}^{M, \gamma}$ be the mild solution of the equation

$$
\left\{\begin{array}{l}
d \hat{\mathcal{Y}}_{t}^{M, \gamma}=A \hat{\mathcal{Y}}_{t}^{M, \gamma} d t+\sum_{i=1}^{\infty} C_{i}(t) \hat{\mathcal{Y}}_{t}^{M, \gamma} d \beta_{t}^{i}+\sum_{i=1}^{M} C_{i}(t)(T-t)^{\alpha} \gamma_{t} d \beta_{t}^{i}, \\
\hat{\mathcal{Y}}_{0}^{M, \gamma}=0 .
\end{array}\right.
$$

Similarly, let $\hat{\mathcal{Y}}^{\gamma}$ be the mild solution of equation of

$$
\left\{\begin{array}{l}
d \hat{\mathcal{Y}}_{t}^{\gamma}=A \hat{\mathcal{Y}}_{t}^{\gamma} d t+\sum_{i=1}^{\infty} C_{i}(t) \hat{\mathcal{Y}}_{t}^{\gamma} d \beta_{t}^{i}+\sum_{i=1}^{\infty} C_{i}(t)(T-t)^{\alpha} \gamma_{t} d \beta_{t}^{i} \\
\hat{\mathcal{Y}}_{0}^{\gamma}=0
\end{array}\right.
$$

We see that equation (4.15) (respectively, (4.16)) coincide with equation (4.4) (respectively, (4.3)) with $s=x=\Gamma=\rho=0, N=\infty$ and $\gamma$ replaced by $(T-\cdot)^{\alpha} \gamma$.

The equality (4.13) reads:

$$
\mathbb{E} \int_{0}^{T}\left\langle S^{M}(l), \gamma_{l}\right\rangle d l=\mathbb{E} \int_{0}^{T}\left\langle(T-l)^{\alpha} \sum_{i=1}^{M} C_{i}^{*}(l) Q_{l} e_{i}, \gamma_{l}\right\rangle d l=\mathbb{E}\left\langle\hat{\mathcal{Y}}_{T}^{M, \gamma}, \eta\right\rangle+\mathbb{E} \int_{0}^{T}\left\langle\hat{\mathcal{Y}}_{l}^{M, \gamma}, f_{l}\right\rangle d l
$$

By the estimate (4.7), we have $\left|\mathbb{E} \int_{0}^{T}\left\langle S^{M}(l), \gamma_{l}\right\rangle d l\right| \leq c \mid \gamma_{L_{\mathcal{P}}^{2}([0, T] \times \Omega, H)}$. Since the set of bounded elements $\gamma$ is dense in $L_{\mathcal{P}}^{2}([0, T] \times \Omega, H)$ it follows that $\left|S^{M}\right|_{L_{\mathcal{P}}^{2}([0, T] \times \Omega, H)} \leq c$. Since, by Theorem 4.3, the right-hand side of (4.17) converges as $M \rightarrow \infty$ (to the limit $\mathbb{E}\left\langle\hat{\mathcal{Y}}_{T}^{\gamma}, \eta\right\rangle+\mathbb{E} \int_{0}^{T}\left\langle\hat{\mathcal{Y}}_{l}^{\gamma}, f_{l}\right\rangle d l$ ) when $\gamma$ is bounded, we conclude that $S^{M}$ converges weakly in $L_{\mathcal{P}}^{2}([0, T] \times \Omega, H)$.

If we replace $Q$ by the approximating operators $Q^{M}$ we obtain the same limit: 
Lemma $4.12(T-\cdot)^{\alpha} \sum_{i=1}^{M} C_{i}^{*}(\cdot) Q^{M} e_{i}$ converges weakly to $(T-\cdot)^{\alpha} \sum_{i=1}^{\infty} C_{i}^{*}(\cdot) Q . e_{i}$ in $L_{\mathcal{P}}^{2}([0, T] \times$ $\Omega, H)$.

Proof. The proof of the existence of the weak limit of the sequence $(T-\cdot)^{\alpha} \sum_{i=1}^{M} C_{i}^{*}(\cdot) Q^{M} e_{i}$ follows the same argument as in the proof of the above Lemma replacing equation (4.15) by

$$
\left\{\begin{array}{l}
d \hat{\mathcal{Y}}_{t}^{M, \gamma}=A \hat{\hat{\mathcal{Y}}}_{t}^{M, \gamma} d t+\sum_{i=1}^{M} C_{i}(t) \hat{\hat{\mathcal{Y}}}_{t}^{M, \gamma} d \beta_{t}^{i}+\sum_{i=1}^{M} C_{i}(t)(T-t)^{\alpha} \gamma_{t} d \beta_{t}^{i}, \\
\hat{\mathcal{Y}}_{0}^{M, \gamma}=0 .
\end{array}\right.
$$

and replacing the second equality in (4.17) by

$$
E \int_{0}^{T}\left\langle(T-l)^{\alpha} \sum_{i=1}^{M} C_{i}^{*}(l) Q_{l}^{M} e_{i}, \gamma_{l}\right\rangle d l=\mathbb{E}\left\langle\hat{\mathcal{Y}}_{T}^{M, \gamma}, \eta\right\rangle+\mathbb{E} \int_{0}^{T}\left\langle\hat{\mathcal{Y}}_{l}^{M, \gamma}, f_{l}\right\rangle d l,
$$

which follows from (4.12). The proof that the limit equals $(T-\cdot)^{\alpha} \sum_{i=1}^{\infty} C_{i}^{*}(\cdot) Q \cdot e_{i}$ comes from the observation that $\mathbb{E} \sup _{s \in[0, T]}\left|\hat{\mathcal{Y}}_{s}^{M, \gamma}-\hat{\mathcal{Y}}_{s}^{\gamma}\right|^{p} \rightarrow 0$, which corresponds to the convergence $\tilde{\mathcal{Y}}^{M, M} \rightarrow \mathcal{Y}$ in Theorem 4.3 .

We are now in a position to prove existence of a solution to the singular BSDE (4.1). For the uniqueness part we need the following Lemma on linear BSDEs with unbounded forcing term which proof will be postponed to the Appendix.

Lemma 4.13 Assume that $\xi$ is a progressively measurable process in $H$ with $\mathbb{E} \int_{0}^{T}(T-l)^{2 \alpha}\left|\xi_{l}\right|^{2} d l<$ $\infty$. Then for any $N \in \mathbb{N}$ and any $\eta \in L^{2}\left(\Omega, \mathcal{F}_{T}, \mathbb{P}\right)$ there exists a unique pair of processes $(p, q)$ with $p$ progressively measurable in $H$, with $s \mapsto p_{s}$ continuous from $[0, T]$ to $L^{2}(\Omega, \mathcal{F}, H)$, and $q \in L_{\mathcal{P}}^{2}\left(\Omega \times[0, T], \mathcal{S}_{2}(H)\right)$ such that:

$$
p_{s}=e^{(T-s) A^{*}} \eta+\int_{s}^{T} e^{(l-s) A^{*}} \sum_{i=1}^{N} C_{i}^{*}(l) q_{l} e_{i} d l+\int_{s}^{T} e^{(l-s) A^{*}} \xi_{l} d l-\sum_{i=1}^{\infty} \int_{s}^{T} e^{(l-s) A^{*}} q_{l} e_{i} d \beta_{l}^{i} .
$$

Moreover, letting $\gamma=\rho=0, M=\infty$ and $\Gamma \in L_{\mathcal{P}}^{\infty}\left(\Omega \times[0, T], \mathcal{S}_{2}(H)\right)$ in equation (4.4) the following duality relation holds:

$$
\mathbb{E}\left\langle p_{s}, x\right\rangle+\mathbb{E} \int_{s}^{T}\left\langle q_{l}, \Gamma_{l}\right\rangle_{\mathcal{S}_{2}(H)} d s=\mathbb{E}\left\langle\tilde{\mathcal{Y}}_{T}^{N}, \eta\right\rangle+\mathbb{E} \int_{s}^{T}\left\langle(T-l)^{-\alpha} \tilde{\mathcal{Y}}_{l}^{N},(T-l)^{\alpha} \xi_{l}\right\rangle d l .
$$

Notice that $\sup _{l \in[s, T]} \mathbb{E}\left|\tilde{\mathcal{Y}}_{l}^{N}\right|^{2}<+\infty$ and that $\alpha<1 / 2$, so the last integral is well defined.

Proof. The proof of this lemma is postponed to the Appendix.

Theorem 4.14 The pair $(P, Q)$ constructed in Corollary 4.8 is the unique mild solution to the singular BSDEs (4.1).

Proof. Existence: As noticed in Remark 4.2, for any fixed $s$, the map $g \mapsto \int_{s}^{T}(T-l)^{-\alpha} e^{(l-s) A^{*}} g_{l} d l$ is weakly continuous from $L_{\mathcal{P}}^{2}([0, T] \times \Omega, H)$ to $L^{2}\left(\Omega, \mathcal{F}_{T}, \mathbb{P}, H\right)$.

Hence, by Lemma 4.11 and Lemma 4.12 the sequence $\sum_{i=1}^{N} \int_{s}^{T} e^{(l-s) A^{*}} C_{i}^{*}(l) Q_{l}^{N} d l$ converges, weakly in $L^{2}\left(\Omega, \mathcal{F}_{T}, \mathbb{P}, H\right)$, to $\sum_{i=1}^{\infty} \int_{s}^{T} e^{(l-s) A^{*}} C_{i}^{*}(l) Q_{l} e_{i} d l$. 
Similarly $\sum_{i=1}^{\infty} \int_{s}^{T} e^{(l-s) A^{*}} Q_{l}^{N} e_{i} d \beta_{l}^{i}$ converges weakly to $\sum_{i=1}^{\infty} \int_{s}^{T} e^{(l-s) A^{*}} Q_{l} e_{i} d \beta_{l}^{i}$, since $Q^{N}$ converges weakly to $Q$ in $L^{2}\left(\Omega \times[0, T], \mathcal{S}_{2}(H)\right)$.

The fact that $(P, Q)$ is a mild solution of the singular BSDEs (4.1) follows by passing to the limit in equation (4.11) .

Uniqueness: Let $(P, Q)$ and $\left(P^{\prime}, Q^{\prime}\right)$ two solutions and let $\bar{P}=P-P^{\prime}$ and $\bar{Q}=Q-Q^{\prime}$. Moreover let $\bar{L}$ be the weak limit in $L_{\mathcal{P}}^{2}(\Omega \times[0, T])$, as $N \rightarrow \infty$, of $(T-\cdot)^{\alpha} \sum_{i=1}^{N} C_{i}^{*}(s) \bar{Q}_{s} e_{i}$ and define:

$$
\bar{\varphi}_{s}^{N}=\bar{L}_{s}-(T-s)^{\alpha} \sum_{i=1}^{N} C_{i}^{*}(s) \bar{Q}_{s} e_{i}
$$

Then equation (4.2) yields:

$$
\bar{P}_{s}=-\sum_{i=1}^{\infty} \int_{s}^{T} e^{(l-s) A^{*}} \bar{Q}_{l} d \beta_{l}^{i}+\sum_{i=1}^{N} \int_{t}^{T} e^{(l-s) A^{*}} C_{i}^{*}(l) \bar{Q}_{l} e_{i} d l+\int_{s}^{T} e^{(l-s) A^{*}}(T-l)^{-\alpha} \bar{\varphi}_{l}^{N} d l .
$$

thus, for any fixed $N,(\bar{P}, \bar{Q})$ is the unique mild solution of equation (4.19) with $\xi_{l}=(T-l)^{-\alpha} \bar{\varphi}_{l}^{N}$. By Lemma 4.13 we obtain that, for all $\mathcal{S}_{2}$-valued bounded predictable $\Gamma$ :

$$
\mathbb{E}\left\langle\bar{P}_{s}, x\right\rangle+\mathbb{E} \int_{s}^{T}\left\langle\bar{Q}_{l}, \Gamma_{l}\right\rangle_{\mathcal{S}_{2}(H)} d l=\mathbb{E} \int_{s}^{T}\left\langle(T-l)^{-\alpha} \tilde{\mathcal{Y}}_{l}^{N, \Gamma}, \bar{\varphi}_{l}^{N}\right\rangle_{L_{2}(H)} d l,
$$

where again $\tilde{\mathcal{Y}}^{N, \Gamma}$ is the mild solution of equation (4.4) with $\gamma=\rho=0$. By Theorem 4.3 we have, in particular: $\sup _{l \in[t, T], N \in \mathbb{N}} \mathbb{E}\left|\tilde{\mathcal{Y}}_{s}^{N, \Gamma}\right|^{2} \leq c$, and $\mathbb{E}\left|\tilde{\mathcal{Y}}_{l}^{N, \Gamma}-\mathcal{Y}_{l}^{\Gamma}\right|^{2} \rightarrow 0, \quad \forall l \in[s, T]$, where $\mathcal{Y}^{\Gamma}$ is the mild solution of equation (4.4) with $\gamma=\rho=0$. In particular $\tilde{\mathcal{Y}}^{N, \Gamma}$ converges strongly to $\mathcal{Y}^{\Gamma}$ in $L_{\mathcal{P}}^{2}(\Omega \times[s, T], H)$.

Since $\bar{\varphi}^{N}$ converges weakly to 0 , we obtain:

$$
\mathbb{E}\left\langle\bar{P}_{s}, x\right\rangle+\mathbb{E} \int_{s}^{T}\left\langle\bar{Q}_{l}, \Gamma_{l}\right\rangle_{L_{2}(H)} d l=0
$$

which concludes the proof of uniqueness.

\subsection{Trace Class Regularity}

We will now prove that the martingale term $Q$ enjoys a trace class regularity that will be essential to formulate the maximum principle.

Proposition 4.15 If $(P, Q)$ is the unique mild solution of equation (4.1), then

$$
\mathbb{E} \int_{0}^{T}(T-l)^{2 \alpha}\left|Q_{l}\right|_{\mathcal{S}_{1}(H)}^{2} d l \leq c\left(\mathbb{E}|\eta|^{2}+\mathbb{E} \int_{0}^{T}\left|f_{l}\right|^{2} d l\right)
$$

where the constant $c$ only depends on the constants in Hypothesis 4.1.

Proof. Since $Q_{l}$ is, $\mathbb{P}$-a.s. of class $\mathcal{S}_{2}(H)$ and therefore compact, it can be written (see Appendix 1) as:

$$
Q_{l}=\sum_{j=1}^{\infty} a_{j}(l) h_{j}(l)\left\langle g_{j}(l), \cdot\right\rangle
$$


where $a_{j}(l) \in \mathbb{R}$ and $\left(h_{j}(l)\right)_{j},\left(g_{j}(l)\right)_{j}$ are orthonormal bases in $H$. Moreover, we can choose the processes $a_{j}(l), h_{j}(l), g_{j}(l)(l \in[0, T])$ to be progressively measurable. Let

$$
\Gamma_{l}^{n}=\varphi(l) \sum_{j=1}^{n} \operatorname{sgn}\left(a_{j}(l)\right) h_{j}(l)\left\langle g_{j}(l), \cdot\right\rangle
$$

where $\varphi$ is an arbitrary positive real-valued bounded progressively measurable process. We note that $\left|\Gamma_{l}^{n}\right|_{\mathcal{L}(H)} \leq \varphi(l)$ and, being of rank $n$, the process $\Gamma^{n}$ is also bounded in $\mathcal{S}_{2}(H)$.

By (4.13) we have

$$
\mathbb{E} \int_{0}^{T}\left\langle Q_{l}, \Gamma_{l}^{n}\right\rangle_{\mathcal{S}_{2}(H)} d l=\mathbb{E}\left\langle\mathcal{Y}_{T}^{n}, \eta\right\rangle+\mathbb{E} \int_{0}^{T}\left\langle\mathcal{Y}_{l}^{n}, f_{l}\right\rangle d l
$$

where $\mathcal{Y}^{n}$ is now the solution to equation (4.3) with $s=x=\gamma=\rho=0$ and $\Gamma=\Gamma^{n}$. If we compute $\left\langle Q_{l}, \Gamma_{l}^{n}\right\rangle_{\mathcal{S}_{2}(H)}$ and we estimate $\mathbb{E}\left|\mathcal{Y}_{T}^{n}\right|^{2}$ by (4.7) and $\mathbb{E} \int_{0}^{T}\left|\mathcal{Y}_{l}^{n}\right|^{2} d l$ by (4.9) we obtain:

$$
\begin{aligned}
\mathbb{E} \int_{0}^{T} \sum_{j=1}^{n}\left|a_{j}(l)\right| \varphi(l) d l \leq & c\left(\mathbb{E}|\eta|^{2}\right)^{\frac{1}{2}}\left(\mathbb{E} \int_{0}^{T}(T-l)^{-2 \alpha} \varphi(l)^{2} d l\right)^{\frac{1}{2}} \\
& +c\left(\int_{0}^{T} \mathbb{E}\left|f_{l}\right|^{2} d l\right)^{\frac{1}{2}}\left(\mathbb{E} \int_{0}^{T} \varphi(l)^{2} d l\right)^{\frac{1}{2}} .
\end{aligned}
$$

If now $n \rightarrow \infty$, recalling that $\sum_{j=1}^{\infty}\left|a_{j}\right|=|Q|_{\mathcal{S}_{1}(H)}$ (see Appendix 1 below) we get:

$$
\mathbb{E} \int_{0}^{T} \varphi(l)\left|Q_{l}\right|_{\mathcal{S}_{1}} d l \leq c_{f, \eta}^{\frac{1}{2}}\left(\mathbb{E} \int_{0}^{T}(T-l)^{-2 \alpha} \varphi(l)^{2} d l\right)^{\frac{1}{2}},
$$

where $c_{f, \eta}=c\left(\mathbb{E}|\eta|^{2}+\mathbb{E} \int_{0}^{T}\left|f_{l}\right|^{2} d l\right)$. Denoting $\tilde{\varphi}(l)=(T-l)^{-\alpha} \varphi(l)$, we can rewrite the last estimate as:

$$
\mathbb{E} \int_{0}^{T} \tilde{\varphi}(l)\left[(T-l)^{\alpha}\left|Q_{l}\right|_{L_{1}(H)}\right] d l \leq c_{f, \eta}^{\frac{1}{2}}|\tilde{\varphi}|_{L_{\mathcal{P}}^{2}(\Omega, \times[0, T])}
$$

and the claim follows from the arbitrariness of $\varphi$.

We end this section by proving that, under the following additional assumption, the weak limit that defines the term $\sum_{i=1}^{N} \int_{s}^{T} e^{(l-s) A^{*}} C_{i}^{*}(l) Q_{l} e_{i} d l$ in Definition 4.1 is indeed a strong limit in $L^{1}$.

Hypothesis 4.16 We have $\sum_{i=1}^{\infty}\left|e^{s A^{*}} C_{i}^{*}(l) x\right|^{2} \leq c s^{-2 \alpha}|x|^{2}$ for all $s>0, l \geq 0, x \in H$, with $\alpha<1 / 2$.

Remark 4.17 In the example in Section 2.1 this requirement coincides with Hypothesis 4.1-2 since $A$ and $C_{i}$ are self adjoint.

Proposition 4.18 If Hypothesis 4.16 holds, in addition to Hypothesis 4.1, then the sequence $\sum_{i=1}^{N} \int_{s}^{T} e^{(l-s) A^{*}} C_{i}^{*}(l) Q_{l} e_{i} d l$ converges strongly in $L^{1}\left(\Omega, \mathcal{F}_{T}, \mathbb{P} ; H\right)$ as $N \rightarrow \infty$. The limit obviously coincides with the weak limit in $L^{2}\left(\Omega, \mathcal{F}_{T}, \mathbb{P} ; H\right)$ introduced in Definition 4.1. 
Proof. As above we expand $Q$ as $Q_{l}=\sum_{j=1}^{\infty} a_{j}(l) h_{j}(l)\left\langle g_{j}(l), \cdot\right\rangle$. Then

$$
\begin{aligned}
\sum_{i=1}^{N} \mathbb{E}\left|\int_{s}^{T} e^{(l-s) A^{*}} C_{i}^{*}(l) Q_{l} e_{i} d l\right| & \leq \mathbb{E} \int_{s}^{T} \sum_{i=1}^{N}\left|e^{(l-s) A^{*}} C_{i}^{*}(l) \sum_{j=1}^{\infty} a_{j}(l) h_{j}(l)\left\langle g_{j}(l), e_{i}\right\rangle\right| d l \\
& \leq \mathbb{E} \int_{s}^{T} \sum_{i=1}^{N} \sum_{j=1}^{\infty}\left|a_{j}(l)\right|\left|e^{(l-s) A^{*}} C_{i}^{*}(l) h_{j}(l)\right|\left|\left\langle g_{j}(l), e_{i}\right\rangle\right| d l \\
& \leq \mathbb{E} \int_{s}^{T} \sum_{j=1}^{\infty}\left|a_{j}(l)\right|\left(\sum_{i=1}^{\infty}\left|e^{(l-s) A^{*}} C_{i}^{*}(l) h_{j}(l)\right|^{2}\right)^{\frac{1}{2}}\left(\sum_{i=1}^{\infty}\left\langle g_{j}(l), e_{i}\right\rangle^{2}\right)^{\frac{1}{2}} \\
& \leq c \mathbb{E} \int_{s}^{T} \sum_{j=1}^{\infty}\left|a_{j}(l)\right|(l-s)^{-\alpha} d l \\
& =c \mathbb{E} \int_{s}^{T}\left|Q_{l}\right|_{\mathcal{S}_{1}(H)}(l-s)^{-\alpha} d l \\
& \leq c\left(\int_{s}^{T}\left|Q_{l}\right|_{\mathcal{S}_{1}(H)}^{2}(T-l)^{2 \alpha} d l\right)^{\frac{1}{2}}\left(E \int_{s}^{T}(l-s)^{-2 \alpha}(T-l)^{-2 \alpha} d s\right)^{\frac{1}{2}} .
\end{aligned}
$$

Strong convergence in $L^{1}(\Omega ; H)$ follows from Proposition 4.15. The coincidence of the two limits is evident by testing them against any bounded $\mathcal{F}_{s}$-measurable $H$-valued random variable.

\section{Appendix}

\subsection{Trace Class Operators}

We collect here some basic facts on Hilbert-Schmidt and trace class operators. For a detailed treatment and for the proof of the results stated below see [18].

Given a real separable Hilbert space $H$ we denote by $\mathcal{S}_{2}(H)$ the Hilbert space of Hilbert Schmidt operators $H \rightarrow H$ endowed with the scalar product $\langle L, M\rangle_{\mathcal{S}_{2}(H)}=\sum_{i=1}^{\infty}\left\langle L e_{i}, M e_{i}\right\rangle_{H}$ where $\left(e_{i}\right)$ is any orthonormal basis in $H$.

If $L \in \mathcal{S}_{2}(H)$ then there exists a sequence $\left(a_{j}^{L}\right)_{j \in \mathbb{N}} \in \ell_{2}$ and a pair of orthonormal bases $\left(e_{j}^{L}\right)_{j \in \mathbb{N}}$, $\left(h_{j}^{L}\right)_{j \in \mathbb{N}}$ in $H$ such that $L=\sum_{j=1}^{\infty} a_{j}^{L} h_{j}^{L}\left\langle e_{j}^{L}, \cdot\right\rangle$. Moreover $|L|_{\mathcal{S}_{2}(H)}=\sum_{j=1}^{\infty}\left(a_{j}^{L}\right)^{2}$. Finally if $t \rightarrow$ $L_{t}$ is a $\mathcal{S}_{2}(H)$-valued measurable process then the above objects can be selected with the same measurability property.

We define trace class operators in the following way: $\mathcal{S}_{1}(H)=\left\{L \in \mathcal{S}_{2}(H):|L|_{\mathcal{S}_{1}}<\infty\right\}$, where

$$
|L|_{\mathcal{S}_{1}}:=\sup \left\{\langle B, L\rangle_{\mathcal{S}_{2}}: B \in \mathcal{S}_{2}(H),|B|_{\mathcal{L}(H)} \leq 1\right\} .
$$

The following results are true:

1. If $B \in \mathcal{L}(H)$ and $L \in \mathcal{S}_{1}(H)$ then $L B, B L$ are in $\mathcal{S}_{1}(H)$, and moreover

$$
|L B|_{\mathcal{S}_{1}(H)} \leq|L|_{\mathcal{S}_{1}(H)}|B|_{\mathcal{L}(H)},|B L|_{\mathcal{S}_{1}(H)} \leq|L|_{\mathcal{S}_{1}(H)}|B|_{\mathcal{L}(H)} \text {. }
$$

2. If $L \in \mathcal{S}_{1}(H)$ and $\left(e_{i}\right)$ is an arbitrary orthonormal basis, the trace $\operatorname{Tr}(L):=\sum_{i=1}^{\infty}\left\langle e_{i}, L e_{i}\right\rangle$ converges absolutely and its value is independent of the choice of the basis $\left(e_{i}\right)$. 
3. Using the expansion introduced above $|L|_{\mathcal{S}_{1}(H)}=\sum_{j=1}^{\infty}\left|a_{j}^{L}\right|, \operatorname{Tr}(L)=\sum_{j=1}^{\infty} a_{j}^{L}$ consequently $|\operatorname{Tr}(L)| \leq|L|_{\mathcal{S}_{1}(H)}$.

\subsection{Linear, Infinite-dimensional BSDEs with Unbounded Terms}

We prove here that infinite dimensional BSDEs can be well-posed even if they include an unbounded term. The following proposition is an extension of the results in [12] and its proof follows the same lines.

Proposition 5.1 Under the same assumptions and notations of Section 2, let $\eta \in L^{2}\left(\Omega, \mathcal{F}_{T}, \mathbb{P} ; H\right)$ and let $\phi$ be a progressively measurable process in $H$ satisfying

$$
\mathbb{E} \int_{0}^{T}(T-s)^{2 \alpha}\left|\varphi_{s}\right|^{2} d s<\infty
$$

then the BSDE

$$
-d p_{t}=\left(A^{*} p_{t}+\varphi_{t}\right) d t-q_{t} d W_{t}, \quad p_{T}=\eta
$$

admits a unique mild solution, that is a unique pair of processes $(p, q)$ with $p \in L_{\mathcal{P}}^{2}(\Omega, C([0, T], H))$, $q \in L_{\mathcal{P}}^{2}\left(\Omega \times[0, T], \mathcal{S}_{2}(H)\right)$ verifying

$$
p_{t}=e^{(T-t) A^{*}} \eta+\int_{t}^{T} e^{(l-t) A^{*}} \varphi_{l} d l-\int_{t}^{T} e^{(l-t) A^{*}} q_{l} d W_{l} .
$$

Moreover the following estimate holds:

$$
\mathbb{E} \int_{t}^{T}\left|q_{s}\right|_{\mathcal{S}_{2}(H)}^{2} d s+\sup _{s \in[t, T]} \mathbb{E}\left|p_{s}\right|^{2} \leq c \mathbb{E}|\eta|^{2}+c(T-t)^{1-2 \alpha} \int_{t}^{T}(T-s)^{2 \alpha} \mathbb{E}\left|\varphi_{s}\right|^{2} d s .
$$

Proof. The uniqueness follows directly from [12] since the difference $(\bar{p}, \bar{q})$ of two solutions satisfies:

$$
-d \bar{p}_{t}=A^{*} \bar{p}_{t} d t-\bar{q}_{t} d W_{t}, \quad \bar{p}_{T}=0 .
$$

Concerning existence, let us set:

$$
p_{t}=e^{(T-t) A^{*}} \mathbb{E}\left(\eta \mid \mathcal{F}_{t}\right)+\int_{t}^{T} e^{(s-t) A^{*}} \mathbb{E}\left(\varphi_{s} \mid \mathcal{F}_{t}\right) d s
$$

Moreover, by the martingale representation theorem,

$$
\mathbb{E}\left(\varphi_{s} \mid \mathcal{F}_{t}\right)=\varphi_{s}-\int_{t}^{s} g(s, l) d W_{l}, \quad \mathbb{E}\left(\eta \mid \mathcal{F}_{t}\right)=\eta-\int_{t}^{T} h(l) d W_{l}
$$

Notice that

$$
\mathbb{E} \int_{t}^{\rho}|g(\rho, \sigma)|^{2} d \sigma \leq \mathbb{E}\left|\varphi_{\rho}\right|^{2}+\mathbb{E}\left|\mathbb{E}\left(\varphi_{\rho} \mid \mathcal{F}_{t}\right)\right|^{2} \leq 2 \mathbb{E}\left|\varphi_{\rho}\right|^{2}, \quad \mathbb{E} \int_{t}^{\rho}|h(\sigma)|^{2} d \sigma \leq 2 \mathbb{E}|\eta|^{2} .
$$


From the above two equations, we have:

$$
\begin{aligned}
p_{t}= & \int_{t}^{T} e^{(s-t) A^{*}} \varphi_{s} d s-\int_{t}^{T} e^{(s-t) A^{*}}\left(\int_{t}^{s} g(s, l) d W_{l}\right) d s+e^{(T-t) A^{*}} \eta-\int_{t}^{T} e^{(T-t) A^{*}} h(l) d W_{l} \\
= & \int_{t}^{T} e^{(s-t) A^{*}} \varphi_{s} d s-\int_{t}^{T} e^{(l-t) A^{*}}\left(\int_{l}^{T} e^{(s-l) A^{*}} g(s, l) d s\right) d W_{l} \\
& \quad+e^{(T-t) A^{*}} \eta-\int_{t}^{T} e^{(l-t) A^{*}} e^{(T-l) A^{*}} h(l) d W_{l} .
\end{aligned}
$$

So setting

$$
q_{l}=\int_{l}^{T} e^{(s-l) A^{*}} g(s, l) d s+e^{(T-l) A^{*}} h(l),
$$

we deduce that $(P, Q)$ is the unique solution

Let us now establish the estimates.

$$
\begin{aligned}
\mathbb{E}\left|q_{\sigma}\right|^{2} & \leq c\left(\int_{\sigma}^{T}(T-\rho)^{-\alpha}(T-\rho)^{\alpha} \mathbb{E}|g(\rho, \sigma)| d \rho\right)^{2}+c \mathbb{E}|h(\sigma)|^{2} d \sigma \\
& \leq(T-t)^{1-2 \alpha} \int_{\sigma}^{T}(T-\rho)^{2 \alpha} \mathbb{E}|g(\rho, \sigma)|^{2} d \rho+c \mathbb{E}|h(\sigma)|^{2} d \sigma
\end{aligned}
$$

Thus

$$
\begin{aligned}
\int_{t}^{T} \mathbb{E}\left|q_{\sigma}\right|^{2} d \sigma & \leq(T-t)^{1-2 \alpha} \int_{t}^{T} \int_{\sigma}^{T}(T-\rho)^{2 \alpha} \mathbb{E}|g(\rho, \sigma)|^{2} d \rho d \sigma+c \int_{t}^{T} \mathbb{E}|h(\sigma)|^{2} d \sigma \\
& \leq(T-t)^{1-2 \alpha} \int_{t}^{T}(T-\rho)^{2 \alpha}\left(\int_{t}^{\rho} E|g(\rho, \sigma)|^{2} d \sigma\right) d \rho+c \int_{t}^{T} \mathbb{E}|h(\sigma)|^{2} d \sigma \\
& \leq(T-t)^{1-2 \alpha} \int_{t}^{T}(T-\rho)^{2 \alpha} \mathbb{E}|\varphi(\rho)|^{2} d \rho+c \mathbb{E}|\eta|^{2} .
\end{aligned}
$$

On the other hand,

$$
\begin{aligned}
\mathbb{E}\left|p_{t}\right|^{2} & \leq c \mathbb{E}\left|\int_{t}^{T} \mathbb{E}\left(\varphi_{s} \mid \mathcal{F}_{t}\right) d s\right|^{2}+\mathbb{E}\left|\mathbb{E}\left(\eta \mid \mathcal{F}_{t}\right)\right|^{2}=\mathbb{E}\left[\int_{t}^{T}(T-s)^{-\alpha}(T-s)^{\alpha}\left|\mathbb{E}\left(\varphi_{s} \mid \mathcal{F}_{t}\right)\right| d s\right]^{2}+\mathbb{E}|\eta|^{2} \\
& \leq(T-t)^{1-2 \alpha} \int_{t}^{T}(T-s)^{2 \alpha} \mathbb{E}|\varphi(s)|^{2} d s+\mathbb{E}|\eta|^{2}
\end{aligned}
$$

which concludes the proof.

Proof of Lemma 4.13. Using Proposition 5.1, existence and uniqueness follows by a standard contraction argument (see [12]). The final duality property is established by a simple truncation argument.

\section{References}

[1] A. Bensoussan. Stochastic maximum principle for distributed parameter systems. J. Franklin Inst. 315 (1983), no. 5-6, 387-406. 
[2] G. Da Prato, J. Zabczyk. Stochastic equations in infinite dimensions. Encyclopedia of Mathematics and its Applications, 44. Cambridge University Press, Cambridge, 1992.

[3] G. Da Prato, J. Zabczyk. Ergodicity for Infinite Dimensional Systems. London Mathematical Society Lecture Note Series, 229. Cambridge University Press, Cambridge, 1996.

[4] K. Du, Q. Meng. Stochastic maximum principle for infinite dimensional control systems. Preprint arXiv:1208.0529, 2012.

[5] K. Du, Q. Meng. A maximum principle for optimal control of stochastic evolution equations. SIAM J. Control Optim. 51 (2013), no. 6, 4343-4362.

[6] M. Fuhrman, Y. Hu, G. Tessitore. Stochastic maximum principle for optimal control of SPDEs. Appl. Math. Optim. 68 (2013), no. 2, 181-217.

[7] M. Fuhrman, G. Tessitore. Nonlinear Kolmogorov equations in infinite dimensional spaces: the backward stochastic differential equations approach and applications to optimal control. Ann. Probab. 30 (2002), no. 3, 1397-1465.

[8] G. Guatteri. Stochastic maximum principle for SPDEs with noise and control on the boundary. Systems Control Lett. 60 (2011), no. 3, 198-204.

[9] G. Guatteri, G. Tessitore. On the backward stochastic Riccati equation in infinite dimensions. SIAM J. Control and Optim. 44 (2005), no. 1, 159-194.

[10] D. Henry. Geometric theory of semilinear parabolic equations. Lecture Notes in Mathematics, 840. Springer, Berlin, 1981.

[11] Y. Hu, S. Peng. Adapted solution of a backward semilinear stochastic evolution equation. Stochastic Anal. Appl. 9 (1991), no. 4, 445-459.

[12] Y. Hu, S. Peng. Maximum principle for semilinear stochastic evolution control systems. Stochastics Stochastics Rep. 33 (1990), no. 3-4, 159-180.

[13] A. Lunardi. Analytic semigroups and optimal regularity in parabolic problems. Progress in Nonlinear Differential Equations and their Applications, 16. Birkhauser, Basel, 1995.

[14] Q. Lü, X. Zhang. General Pontryagin-type stochastic maximum principle and backward stochastic evolution equations in infinite dimensions. SpringerBriefs in Mathematices. Springer, Cham, 2014.

[15] B. Øksendal, A. Sulem, T. Zhang. Optimal control of stochastic delay equations and timeadvanced backward stochastic differential equations. Adv. in Appl. Probab. 43 (2011), no. 2, $572-596$.

[16] A. Pazy. Semigroups of linear operators and applications to partial differential equations. Applied Mathematical Sciences, 44. Springer, New York, 1983.

[17] S. Peng. A general stochastic maximum principle for optimal control problems. SIAM J. Control Optim. 28 (1990), no. 4, 966-979. 
[18] J. R. Ringrose. Compact non-self-adjoint operators, Van Nostrand, London, 1971.

[19] S. Tang, X. Li. Maximum principle for optimal control of distributed parameter stochastic systems with random jumps. Differential equations, dynamical systems, and control science, 867-890, Lecture Notes in Pure and Appl. Math., 152, Dekker, New York, 1994.

[20] G. Tessitore. Some remarks on the Riccati equation arising in an optimal control problem with state- and control-dependent noise. SIAM J. Control Optim. 30 (1992), no. 3, 717-744.

[21] G. Tessitore. Existence, uniqueness and space regularity of the adapted solutions of a backward SPDE. Stochastic Anal. Appl. 14 (1996), no 4, 461-486.

[22] J. M. A. M. van Neerven, M. C. Veraar, L. Weis. Stochastic integration in UMD Banach spaces. Ann. Probab. 35 (2007), no. 4, 1438-1478.

[23] J. Yong, X.Y. Zhou. Stochastic controls. Hamiltonian systems and HJB equations. Applications of Mathematics (New York), 43. Springer, New York, 1999.

[24] X.Y. Zhou. On the necessary conditions of optimal controls for stochastic partial differential equations. SIAM J. Control Optim. 31 (1993), no. 6, 1462-1478. 